ThE Astrophysical Journal, 496:808-826, 1998 April 1

(C) 1998. The American Astronomical Society. All rights reserved. Printed in U.S.A.

\title{
THE AGES AND ABUNDANCES OF A LARGE SAMPLE OF M87 GLOBULAR CLUSTERS ${ }^{1}$ \\ Judith G. COHEN, ${ }^{2}$ JOHN P. BLAKESLEE, ${ }^{2}$ AND ANTON RYZHOV ${ }^{2,3}$ \\ Received 1997 September 19; accepted 1997 November 5
}

\begin{abstract}
A subset of 150 globular clusters (GCs) in M87 has been selected for abundance and age determinations from the 1997 sample of Cohen \& Ryzhov. This has been done solely on the basis of the signalto-noise ratios of the spectra. Indices that measure the strength of the strongest spectral features were determined for the M87 GCs and from new data for twelve galactic GCs. Combining the new and existing data for the galactic GCs and comparing the $(U-R)$ colors and the line indices gave qualitative indications for the ages and abundances of the M87 GC system.

Quantitative results, which confirm and extend the qualitative ones, were obtained by applying the Worthey models for the integrated light of stellar systems of a single age, calibrated by observations of galactic globular clusters, to deduce abundances and ages for the objects in our sample.

We find that the M87 GCs span a wide range in metallicity, from very metal-poor to somewhat above solar metallicity. The mean $[\mathrm{Fe} / \mathrm{H}]$ of $-0.95 \mathrm{dex}$ is higher than that of the galactic $\mathrm{GC}$ system, and there is a metal-rich tail that reaches to higher $[\mathrm{Fe} / \mathrm{H}]$ than one finds among the galactic GCs. Excluding the very metal-rich tail, there is marginal evidence for a bimodal distribution over the single one at the $89 \%$ significance level. The two "subpopulations" in this model are located at -1.3 and -0.7 dex and contain $40 \%$ and $60 \%$ of the total, respectively. The dispersion in $[\mathrm{Fe} / \mathrm{H}]$ for each of the model subpopulations is $\sigma=0.3$ dex.

The mean metallicity of the M87 GC system is about a factor of 4 lower than that of the M87 stellar halo at a fixed projected radius $R$. The metallicity inferred from the X-ray studies is similar to that of the M87 stellar halo, not to that of the M87 GC system.

We infer the relative abundances of $\mathrm{Na}, \mathrm{Mg}$, and $\mathrm{Fe}$ in the M87 GCs from the strength of their spectral features. The behavior of these elements between the metal-rich and metal-poor M87 GCs is similar to that shown by the galactic GCs and by halo stars in the Galaxy. The pattern of chemical evolution in these disparate old stellar systems is, as far as we can tell, identical.

Superposed on a very large dispersion in abundance at all $R$, there is a small but real radial gradient in the mean abundance of the M87 GCs with $R$, but no detectable change in the $\mathrm{H} \beta$ index with $R$.

We obtain a median age for the M87 GC system of $13 \mathrm{Gyr}$, similar to that found for the galactic GCs. The dispersion about that value ( $\sigma=2 \mathrm{Gyr}$ ) is small.

Subject headings: galaxies: abundances — galaxies: evolution — galaxies: individual (M87) galaxies: star clusters - stars: abundances
\end{abstract}

\section{INTRODUCTION}

This is the second in a series of papers on the M87 globular cluster system. In Cohen \& Ryzhov (1997; hereafter, Paper I) a sample of M87 globular clusters (GCs) was isolated from the Strom et al. (1981) photographic survey for globular cluster candidates in M87, supplemented by additional candidates in the core of M87 identified in Paper I, and radial velocities were presented for about 300 of these candidates from low-resolution imaging spectrograph (LRIS)/Keck spectra in the slitmask mode. Based on their radial velocities, 205 objects from this sample were selected that are believed to be bona fide M87 GCs. In Paper I we explored the dynamics of this rich GC system, focusing on such issues as the spatial distribution of the M87 GCs and of the M87 halo light, on the velocity dispersion as a function of projected radius, on the deprojections needed to derive the enclosed $M(r)$, and on the $M / L$ ratio as a function of $r$.

\footnotetext{
${ }^{1}$ Based in large part on observations obtained at the W. M. Keck Observatory, which is operated jointly by the California Institute of Technology and the University of California.

2 Palomar Observatory, Mail Stop 105-24, California Institute of Technology, Pasadena, CA 91125.

${ }^{3}$ Current address: Department of Physics, Box 351560, University of Washington, Seattle, WA 98195-1560.
}

In this paper we turn to the inference of abundance and age for the M87 GCs. There have been many efforts along these lines in the past, but because these objects are so faint, most such studies have involved broadband or narrowband photometry of various kinds (Strom et al. 1981; Cohen 1988; Couture, Harris, \& Allbright 1990; Lee \& Geisler 1993). This is a trade-off of desired sample size with desired accuracy of and detail in the results and also ease of calibration of the results. Until the advent of very large groundbased telescopes, the photometric surveys were clearly at an advantage.

Imaging with Hubble Space Telescope (HST) has offered unprecedented photometric accuracy for faint objects such as the M87 GCs, and although the field of view is very small (meaning that spatial gradients within the halo of M87 cannot be followed), many interesting issues have been pursued (Whitmore et al. 1995; Elson \& Santiago 1996a, 1996b). The major new result that has emerged from this work is a strong demonstration of a bimodal color distribution, first suggested in the ground-based work of Lee \& Geisler (1993). We will attempt to verify that here and to discern its origin.

Spectroscopic efforts began with Racine, Oke, \& Searle (1978) and Hanes \& Brodie (1986), who succeeded in reaching only six of the brightest M87 GCs at very low 
resolution. As instrumentation improved, efforts continued at Palomar and at the Multiple Mirror Telescope (Mould, Oke, \& Nemec 1987; Mould et al. 1990; Brodie \& Huchra 1991) at moderate spectral resolution to produce a total sample of about 45 observed objects believed to be M87 GCs, but many of their spectra are of quite low signal-tonoise ratio $(\mathrm{S} / \mathrm{N})$ and suitable only for radial velocity determinations.

Our work takes advantage of the large collecting area of the Keck I $10 \mathrm{~m}$ Telescope and of an efficient multiobject spectrograph, LRIS (Oke et al. 1995). We have assembled a statistically significant sample of object with high-precision spectra, and thus will be able to deduce relatively precise abundances and ages for the M87 GCs.

\section{THE SAMPLE OF M87 GLOBULAR CLUSTERS}

The sample of objects under consideration here is the sample of 205 objects believed to be M87 GCs isolated in Paper I. We use only the spectra taken in the 5200 Å region; we do not use the spectra taken in the $8500 \AA$ region due to the excessive detector noise present in the LRIS at the time (winter 1994) that the redder spectra were taken.

An accurate $v_{r}$ can be obtained from noisier spectra than are required for accurate abundance analyses because of the multiplexing of lines in cross-correlation $v_{r}$ measurements and the characterization of the entire spectrum by only a single parameter. To avoid introducing excessive noise into the measurements, correlations, and analysis presented below, we restrict the sample based on the brightness of an object as viewed through the LRIS slit in these LRIS multislit spectra. The final sample consists of those objects whose continuum level is such (with respect to the background from the night sky and from the halo of M87 itself seen in the particular slitlet of a multislit mask corresponding to the object) that a S/N of 22 or greater per pixel $(1.24 \AA$ pixel $^{-1}$ ) is achieved in the continuum. This is calculated over the region from 5450 to $5500 \AA$ assuming that the spectrum is summed over 8 pixels along the slit, where the scale is 4.7 pixels $\operatorname{arcsec}^{-1}$. (The actual height along the slit extracted during the reduction of each spectrum varied due to variations in the seeing.) Since the readout noise is small, we evaluate this for the sum of all spectra of a given object taken with a given slitmask here, normally two or three $3000 \mathrm{~s}$ exposures. We ignore the effects of cosmic rays in this calculation. For future reference this value is denoted as “QSNR.”

An object may get into the sample on the basis of a single spectrum with $Q S N R \geq 22$. If there are two such spectra for an object, they provide independent measurements. In two cases, sums of two spectra of a particular object taken with different slitmasks met the requirement for $Q S N R$ even though individually each is too noisy.

This test is still not sufficient to produce a sample whose minimum $\mathrm{S} / \mathrm{N}$ is uniform, due to the variation in the placement of objects within the individual slits. If an object is very close to the edge of a slitlet in the slitmask, sky subtraction is much harder, and the $\mathrm{S} / \mathrm{N}$, particularly in areas with strong night-sky emission, may be adversely affected. However, as long as our selection criteria are independent of the strength of the absorption features in the spectra, they should suffice for present purposes.

Two objects (Strom 714 and Strom 1067) were rejected because the appearance of their spectra (not in terms of the spectral features, but rather the one-dimensional spatial profile along the slit) indicated that the objects were not single point sources, but rather slightly extended, or a close pair of point sources.

We end up with a sample for the abundance analysis containing 162 spectra of 150 M87 GCs. The fraction of objects rejected through failure to meet the minimum $\mathrm{S} / \mathrm{N}$ criterion is small, $\approx 6 \%$.

\section{DATA ANALYSIS}

\subsection{Adopted Indices}

There are many systems of indices designed to characterize the strength of the most prominent absorption features in stellar spectra. We have adopted the Lick indices as defined by Burstein et al. (1984), Faber et al. (1985), and Gorgas et al. (1993) for our work on the M87 GCs because of the extensive database in the literature in this system. Measured indices for large numbers of galactic stars are described in Faber et al. (1985) and Gorgas et al. (1993). Indices in this system are given for 17 galactic GCs and 19 GCs in M31 by Burstein et al. (1984). Brodie \& Huchra (1990) present indices in the Lick system for additional M31 and galactic globular clusters, while Burstein et al. (1984) present indices for a sample of nearby galaxies. Worthey (1994) has carried out extensive modeling combining the fluxes predicted by grids of theoretical stellar atmospheres (i.e., Kurucz 1993) with a set of evolutionary tracks to predict semiempirical indices for the integrated light of single age burst models of composite stellar systems of varying metallicity. Chavez, Malgnini, \& Morossi (1995) also attempted to calibrate the Lick indices for the $\mathrm{Mg}$ region.

Lick indices are not defined using fluxed spectra. The original spectra were all taken with the IDS (Robinson \& Wampler 1972), a detector in use at Lick for many years, and were normalized to observations of a quartz lamp. It is thus difficult to reproduce at present the continuum slope of this data without additional information.

Our M87 GC spectra are given as a function of data numbers $\left(2 e^{-1} \mathrm{DN}^{-1}\right)$ versus wavelength $(\lambda)$ rather than flux as a function of wavelength. They can not easily nor accurately be converted into flux because of variable light loss in the LRIS slits due to alignment errors and because some of the nights during which spectra were obtained were not photometric. In addition, as described in Paper I, two gratings with the same dispersion but different blaze angles were used during the 15 month period over which these observations were made.

One therefore has to be careful of the effect of global continuum shape on the measured line indices. Under such circumstances narrow indices should be more reproducible, and we have therefore favored the narrower indices whenever possible. Thus of the three $\mathrm{Mg}$ indices defined in the Lick system, we use $\mathrm{Mg} b$. It measures only the $\mathrm{Mg}$ triplet, while $\mathrm{Mg}_{1}$ and $\mathrm{Mg}_{2}$ also include the $\mathrm{MgH}$ band. The M87 GCs are not of such a late type that the $\mathrm{MgH}$ band is very strong, so use of the $\mathrm{Mg} b$ index should suffice. Measurements of the $\mathrm{Mg}_{1}$ and $\mathrm{Mg}_{2}$ indices show a systematic offset with respect to the Lick measurements, presumably due to differences in continuum slope. All trends described here with respect to $\mathrm{Mg} b$ are also obeyed by the $\mathrm{Mg}_{1}$ and $\mathrm{Mg}_{2}$ indices.

Worthey \& Ottaviani (1997) discuss transformation of measured indices to the Lick system, emphasizing spectral 
resolution and velocity dispersion effects. However, since our spectral resolution is $8 \AA$, while that of the Lick IDS was 8-10 Å depending somewhat on wavelength, we do not need to smooth our spectra to match. No correction for internal velocity dispersion was made either. Unlike the case of galaxies, the velocity dispersion inside GCs is small enough that this is not necessary.

Since there was no Lick index for $\mathrm{H} \alpha$, we defined one. The bandpasses for the adopted indices are listed in Table 1.

\subsection{Data Analysis}

As described in Paper I, all the spectra were reduced using Figaro (Shortridge 1988) scripts written by A. Ryzhov. It became clear that some improvements could be made particularly in the area of cosmic-ray removal and extraction of the final spectrum. About 80 spectra were rereduced using improved scripts and a procedure that required more manual intervention but produced spectra with significantly reduced noise. The spectra selected for rereduction were those at the faint end of the sample and those just below the cutoff in the hopes that a rereduction would get them into the sample.

The $v_{r}$ adopted (all the bandpasses used to compute the indices are of course shifted by the appropriate factor for the $v_{r}$ of each object) are those of Paper I. In the course of the rereduction of the spectra, we noticed five objects whose measured $v_{r}$ appeared to be significantly $\left(\geq 100 \mathrm{~km} \mathrm{~s}^{-1}\right)$ different from the value published in Paper I. Also, the spectrum of one object was not correctly reduced initially. It was not included in Paper I but now has a definite $v_{r}$ indicating membership in the M87 GC system and a S/N such that it is included in the abundance sample. The corrected $v_{r}$ for these five objects and the new $v_{r}$ for Strom 682 are given in Appendix A.

Two of the M87 GCs (Strom 519 and Strom 750) showed emission at the [O III] doublet at 4959 and $5007 \AA$. The emission was clearly extended several arcseconds beyond the cluster in the case of Strom 750, and emission at $\mathrm{H} \alpha$ was also seen. This emission is assumed to originate in the filaments near the nucleus of M87 and is subsequently ignored.

These are faint objects, and even with the best possible algorithm for the removal of the night-sky features, there are often strong residuals of the $\mathrm{NaD}$ lines in the terrestrial atmosphere. Since the radial velocity of an object in M87 is by definition at least $250 \mathrm{~km} \mathrm{~s}^{-1}$, and $v_{r}$ for M87 itself is $1277 \mathrm{~km} \mathrm{~s}^{-1}$, each of the spectra has been inspected by eye, and when necessary, an interpolation of the continuum has been made to remove the residual night-sky emission. Such a procedure was necessary and has been done for almost all of these spectra.

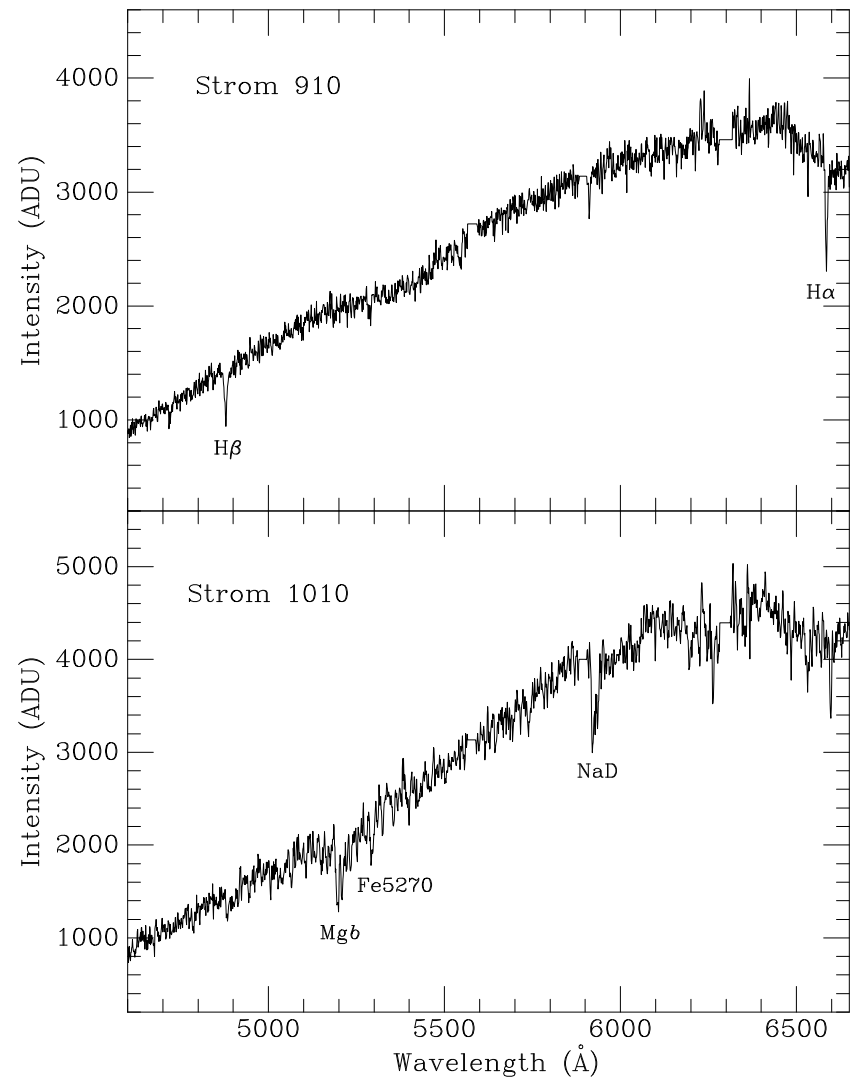

FIG. 1.-A comparison of the spectrum of a metal-poor GC in M87 (Strom 910) with that of a metal-rich one (Strom 1010). Both of these spectra have $Q S N R \approx 40$.

Figure 1 shows a comparison of the spectrum of a relatively high (Strom $1010,[\mathrm{Fe} / \mathrm{H}]_{Z}=-0.2 \mathrm{dex}$ ) and relatively low metallicity GC (Strom $910,[\mathrm{Fe} / \mathrm{H}]_{Z}=-2.2$ dex) in M87 over the regime from 4600 to $6650 \AA$. Both of these objects have $Q S N R \approx 40$. The residuals of the three strongest night-sky features in this spectral region (at 5577, 5891, and $6300 \AA$ A) have been removed via interpolation. The strongest features are identified. Note the relative prominence of the Balmer lines in the low-metallicity object and the much stronger metallic absorption features in the highmetallicity M87 GC.

Because these are spectra taken with a multislit instrument, the wavelength range of the spectrum of a particular object depends on the position of the object in the telescope focal plane. This means that the reddest and bluest features analyzed $(\mathrm{H} \beta$ and $\mathrm{H} \alpha)$ may not be present on all the spectra.

TABLE 1

BANDPASSES FOR ABSORPTION INDICES

\begin{tabular}{|c|c|c|c|c|}
\hline Index & $\begin{array}{c}\text { Blue Continuum } \\
\text { (Å) }\end{array}$ & $\begin{array}{c}\text { Feature Bandpass } \\
(\AA)\end{array}$ & $\begin{array}{c}\text { Red Continuum } \\
\text { (Å) }\end{array}$ & Type \\
\hline $\mathrm{H} \beta$ & $4829.50-4848.25$ & $4849.50-4877.00$ & $4878.25-4892.00$ & EW \\
\hline $\mathrm{Mg}_{1}$ & $4897.00-4958.25$ & $5071.00-5134.75$ & $5303.00-5366.75$ & Mag \\
\hline $\mathbf{M g}_{2}$ & $4897.00-4958.25$ & $5156.00-5197.25$ & $5303.00-5366.75$ & Mag \\
\hline $\operatorname{Mg} b \ldots \ldots \ldots$ & $5144.50-5162.00$ & $5162.00-5193.25$ & $5193.25-5207.00$ & $\mathrm{EW}$ \\
\hline $\mathrm{Fe} \lambda 5270 \ldots \ldots$ & $5235.50-5248.00$ & $5248.00-5286.75$ & $5288.00-5319.25$ & EW \\
\hline $\mathrm{Fe} \lambda 5335 \ldots \ldots$ & $5307.25-5317.25$ & $5314.75-5353.50$ & $5356.00-5364.75$ & EW \\
\hline $\mathrm{NaD}$. & $5863.00-5876.75$ & $5879.25-5910.50$ & $5924.50-5949.25$ & EW \\
\hline $\mathrm{TiO}_{1}$ & $5819.00-5850.25$ & $5939.00-5995.25$ & $6041.00-6104.75$ & Mag \\
\hline $\mathrm{H} \alpha \ldots \ldots$ & $6420.00-6455.00$ & $6548.00-6578.00$ & $6600.00-6640.00$ & EW \\
\hline
\end{tabular}


TABLE 2

INDEX MEASUREMENTS FOR M87 Globular CluSTERS

\begin{tabular}{|c|c|c|c|c|c|c|c|c|c|c|c|}
\hline Identity & $\begin{array}{l}\mathrm{H} \beta \\
(\AA)\end{array}$ & $\begin{array}{c}\mathrm{Mg}_{1} \\
(\mathrm{mag})\end{array}$ & $\begin{array}{c}\mathrm{Mg}_{2} \\
\text { (mag) }\end{array}$ & $\underset{(\AA)}{\operatorname{Mg} b}$ & $\begin{array}{c}\mathrm{Fe} \lambda 5270 \\
(\AA)\end{array}$ & $\begin{array}{c}\mathrm{Fe} \lambda 5335 \\
(\AA)\end{array}$ & $\begin{array}{c}\mathrm{NaD} \\
(\AA)\end{array}$ & $\begin{array}{l}\mathrm{TiO}_{1} \\
\text { (mag) }\end{array}$ & $\begin{array}{l}\mathrm{H} \alpha \\
(\AA)\end{array}$ & $Q S N R$ & $\begin{array}{c}{[\mathrm{Fe} / \mathrm{H}]_{Z}} \\
(\text { dex })\end{array}$ \\
\hline 5001. & 1.02 & -0.003 & 0.090 & 2.38 & 0.96 & 1.56 & 1.14 & 0.021 & 0.58 & 36.2 & -0.95 \\
\hline 5002. & 2.32 & 0.035 & 0.085 & 2.04 & 1.89 & 1.74 & 0.40 & 0.004 & 1.89 & 25.7 & -1.22 \\
\hline 5012. & 2.01 & 0.145 & 0.306 & 5.06 & 3.25 & 3.02 & 5.88 & 0.040 & 1.81 & 23.0 & +0.11 \\
\hline 5015. & 2.61 & 0.021 & 0.089 & 1.70 & 1.24 & 0.96 & 1.53 & -0.013 & 2.72 & 53.9 & -1.14 \\
\hline $5020 \ldots \ldots$ & 1.77 & 0.014 & 0.081 & 1.17 & 2.11 & 1.09 & 1.76 & -0.015 & $\ldots$ & 22.1 & -1.14 \\
\hline $5021 \ldots \ldots$ & 0.89 & 0.081 & 0.220 & 4.13 & 2.22 & 1.43 & 3.73 & 0.023 & 1.78 & 24.3 & -0.23 \\
\hline $5024 \ldots \ldots$ & 2.61 & 0.023 & 0.065 & 1.38 & 0.99 & 1.07 & 1.02 & 0.007 & $\ldots$ & 50.8 & -1.41 \\
\hline $5025 \ldots \ldots$ & 1.72 & 0.024 & 0.094 & 2.55 & 1.47 & 1.63 & 1.28 & 0.024 & $\ldots$ & 24.2 & -0.83 \\
\hline $5026 \ldots \ldots$ & 1.67 & 0.033 & 0.103 & 2.86 & 1.41 & 0.55 & 0.75 & 0.013 & 2.13 & 51.0 & -0.91 \\
\hline 5028. & 1.73 & 0.129 & 0.324 & 5.42 & 3.03 & 2.71 & 6.35 & 0.046 & 1.12 & 56.9 & +0.11 \\
\hline $58 \ldots \ldots \ldots$ & 1.54 & -0.026 & -0.006 & 0.53 & 0.43 & 1.21 & 0.60 & -0.030 & 1.64 & 46.3 & -2.13 \\
\hline $66 \ldots$ & 2.06 & -0.037 & 0.017 & 1.73 & 1.26 & 1.00 & 1.51 & 0.007 & 3.52 & 33.9 & -1.10 \\
\hline $101 \ldots \ldots$ & 1.28 & -0.033 & 0.056 & 1.64 & 0.32 & 0.37 & 1.39 & 0.015 & 2.08 & 26.7 & -1.33 \\
\hline $107 \ldots \ldots$ & 1.41 & -0.008 & 0.113 & 3.23 & 2.12 & 1.73 & 2.35 & 0.025 & 2.98 & 44.4 & -0.53 \\
\hline $141 \ldots \ldots$ & 1.94 & 0.004 & 0.067 & 2.23 & 2.44 & 1.58 & 2.51 & 0.021 & 1.72 & 46.8 & -0.72 \\
\hline $176 \ldots \ldots$ & 1.67 & 0.010 & 0.150 & 2.54 & 1.80 & 1.77 & 1.53 & 0.015 & 2.45 & 33.7 & -0.80 \\
\hline 177. & 1.94 & -0.048 & 0.112 & 2.88 & 2.43 & 2.39 & 0.70 & 0.033 & 3.21 & 30.9 & -0.80 \\
\hline 186. & 2.17 & -0.035 & 0.071 & 1.71 & 0.71 & 0.62 & 1.17 & 0.012 & 2.98 & 37.9 & -1.29 \\
\hline 191. & 1.26 & -0.024 & -0.008 & 1.51 & 1.06 & 0.22 & 0.81 & -0.005 & 1.18 & 27.6 & -1.48 \\
\hline $248 \ldots \ldots$. & 2.36 & -0.026 & 0.086 & 2.22 & 2.42 & 1.45 & 1.50 & 0.011 & 3.05 & 51.3 & -0.87 \\
\hline 279 . & 1.55 & -0.026 & 0.077 & 2.45 & 1.53 & 1.59 & 1.48 & 0.020 & 2.67 & 68.6 & -0.83 \\
\hline 280 . & 2.19 & 0.060 & 0.159 & 3.19 & 2.03 & 0.71 & 1.45 & 0.019 & 0.85 & 31.5 & -0.68 \\
\hline $290 \ldots \ldots \ldots$ & 1.70 & 0.066 & 0.201 & 3.68 & 2.21 & 1.78 & 1.06 & 0.038 & 1.56 & 37.9 & -0.61 \\
\hline $292 \ldots \ldots$. & 0.95 & -0.009 & 0.042 & 2.13 & 0.69 & -0.13 & 1.59 & -0.003 & $\ldots$ & 26.9 & -1.10 \\
\hline $307 \ldots \ldots$ & 2.76 & -0.038 & 0.059 & 1.85 & 2.02 & 1.37 & 1.49 & 0.008 & 2.89 & 41.4 & -0.99 \\
\hline $311 \ldots \ldots$ & 1.38 & -0.023 & 0.108 & 3.03 & 1.31 & 2.17 & 1.26 & 0.034 & 2.53 & 28.1 & -0.72 \\
\hline $313 \ldots \ldots$ & 0.33 & -0.035 & 0.062 & 2.84 & 1.77 & 0.67 & 0.55 & 0.014 & 1.69 & 22.3 & -0.91 \\
\hline $314 \ldots \ldots$. & 1.88 & 0.016 & 0.146 & 4.15 & 2.69 & 2.92 & 2.69 & 0.047 & 1.96 & 65.8 & -0.34 \\
\hline $321 \ldots \ldots$ & 2.36 & -0.073 & -0.028 & 0.96 & 1.38 & 0.26 & 0.73 & -0.003 & 3.24 & 62.3 & -1.75 \\
\hline $323 \ldots \ldots$ & 2.62 & -0.060 & -0.049 & 0.31 & 1.60 & 0.93 & 0.52 & -0.019 & 2.67 & 43.2 & -2.13 \\
\hline $324 \ldots \ldots \ldots$ & 1.09 & -0.019 & 0.091 & 2.76 & 1.90 & 1.28 & 1.62 & 0.002 & 2.84 & 34.5 & -0.76 \\
\hline $330 \ldots \ldots$ & 2.41 & 0.009 & 0.123 & 3.06 & 2.68 & 1.95 & 1.85 & 0.027 & 2.45 & 33.6 & -0.61 \\
\hline $348 \ldots \ldots$ & 2.10 & -0.030 & 0.009 & 1.43 & 2.00 & 1.35 & 1.07 & 0.010 & 1.86 & 83.6 & -1.25 \\
\hline $350 \ldots \ldots$ & 2.79 & -0.035 & 0.026 & 2.30 & 1.83 & 1.58 & 0.75 & 0.005 & 3.07 & 36.6 & -1.02 \\
\hline $376 \ldots \ldots$ & 2.84 & -0.062 & -0.027 & 1.18 & 0.86 & 1.13 & 0.83 & 0.010 & 3.33 & 52.6 & -1.52 \\
\hline $417 \ldots \ldots$ & 1.60 & 0.003 & 0.140 & 3.04 & 2.12 & 1.76 & 1.97 & 0.028 & 2.56 & 109.9 & -0.61 \\
\hline $418 \ldots \ldots \ldots$ & 2.02 & -0.051 & -0.026 & 1.26 & 0.79 & 0.85 & 1.30 & 0.014 & 2.67 & 38.3 & -1.41 \\
\hline $421 \ldots \ldots$ & 1.86 & 0.020 & 0.184 & 3.13 & 2.92 & 2.52 & 3.12 & 0.026 & $\ldots$ & 50.1 & -0.42 \\
\hline $423 \ldots \ldots$ & 2.58 & 0.050 & 0.176 & 3.98 & 2.12 & 2.07 & 2.94 & 0.025 & 1.87 & 45.0 & -0.34 \\
\hline $442 \ldots \ldots$. & 1.93 & -0.038 & 0.007 & 1.67 & 1.82 & 1.26 & 1.10 & 0.014 & 2.83 & 61.5 & -1.18 \\
\hline $453 \ldots \ldots$. & 2.97 & -0.038 & 0.070 & 1.60 & 2.54 & 1.52 & 1.80 & 0.008 & 3.39 & 33.3 & -0.99 \\
\hline $490 \ldots \ldots$. & 1.41 & 0.094 & 0.262 & 5.29 & 3.22 & 3.01 & 6.77 & 0.043 & 2.10 & 70.3 & +0.11 \\
\hline $491 \ldots \ldots$ & 1.83 & -0.036 & 0.019 & 1.03 & 1.84 & 0.58 & 0.83 & 0.019 & 1.99 & 47.2 & -1.56 \\
\hline $492 \ldots \ldots$ & 2.69 & -0.000 & 0.131 & 2.64 & 2.23 & 1.80 & 2.60 & 0.006 & 2.46 & 24.3 & -0.61 \\
\hline $519 \ldots \ldots$ & 1.49 & 0.012 & 0.127 & 3.06 & 3.04 & 0.26 & 2.15 & 0.023 & 1.68 & 29.2 & -0.61 \\
\hline $526 \ldots \ldots$ & 0.73 & 0.038 & 0.194 & 4.45 & 3.07 & 0.59 & 3.53 & 0.021 & $\ldots$ & 44.0 & -0.23 \\
\hline $537 \ldots \ldots$ & 2.98 & -0.048 & 0.033 & 2.06 & 1.98 & -0.48 & 0.49 & 0.036 & 3.08 & 23.0 & -1.37 \\
\hline $571 \ldots \ldots$ & 1.56 & -0.011 & 0.102 & 2.70 & 2.14 & 2.14 & 1.38 & 0.007 & 3.04 & 29.8 & -0.76 \\
\hline $579 \ldots \ldots$ & 2.37 & 0.011 & 0.034 & 0.81 & 0.57 & 0.74 & 0.82 & 0.009 & $\ldots$ & 40.9 & -1.90 \\
\hline $581 \ldots \ldots$ & 1.88 & 0.020 & 0.094 & 1.79 & 1.67 & 2.38 & 1.69 & 0.024 & 2.23 & 43.0 & -0.95 \\
\hline $588 \ldots \ldots$. & 2.22 & -0.057 & 0.043 & 1.92 & 1.44 & 1.01 & 1.24 & 0.003 & 3.12 & 53.1 & -1.10 \\
\hline $602 \ldots \ldots$ & 1.07 & -0.030 & 0.077 & 2.86 & 2.13 & 1.59 & 1.62 & 0.018 & 2.79 & 46.8 & -0.72 \\
\hline $611 \ldots \ldots$ & 2.74 & -0.014 & 0.087 & 1.01 & 1.37 & 1.41 & 0.44 & -0.001 & $\ldots$ & 37.0 & -1.59 \\
\hline $614 \ldots \ldots$. & 3.89 & -0.026 & 0.061 & 1.85 & 1.96 & 0.19 & 1.13 & 0.011 & 2.72 & 25.1 & -1.18 \\
\hline $647 \ldots \ldots$. & 2.10 & 0.025 & 0.137 & 2.10 & 2.39 & 1.59 & 1.55 & 0.009 & $\ldots$ & 51.9 & -0.87 \\
\hline $649 \ldots \ldots$ & 2.04 & -0.043 & 0.005 & 1.55 & 1.47 & 1.31 & 0.99 & 0.005 & 3.09 & 36.2 & -1.29 \\
\hline $651 \ldots \ldots$ & 1.50 & 0.123 & 0.304 & 5.19 & 2.29 & 2.54 & 4.83 & 0.037 & $\ldots$ & 25.9 & +0.04 \\
\hline $664 \ldots \ldots$. & 1.50 & 0.033 & 0.112 & 2.15 & 2.26 & 1.56 & 2.23 & 0.032 & 2.09 & 24.6 & -0.76 \\
\hline $672 \ldots \ldots$ & 2.19 & -0.014 & 0.038 & 1.26 & 0.89 & 0.88 & 0.85 & -0.013 & 2.97 & 52.4 & -1.52 \\
\hline $679 \ldots \ldots$ & 0.59 & 0.011 & 0.150 & 3.66 & 2.54 & 1.53 & 3.00 & 0.034 & 2.49 & 36.1 & -0.38 \\
\hline $680 \ldots \ldots$ & 2.43 & -0.044 & 0.037 & 0.74 & 1.52 & 1.56 & 1.18 & 0.003 & 2.57 & 40.9 & -1.48 \\
\hline $682 \ldots \ldots$ & 2.22 & -0.047 & 0.027 & 1.15 & 1.29 & -0.05 & 0.30 & 0.005 & 2.67 & 30.2 & -1.75 \\
\hline $697 \ldots \ldots$ & 3.21 & 0.046 & 0.159 & 4.88 & 4.01 & 3.26 & 2.40 & 0.035 & 2.32 & 23.1 & -0.27 \\
\hline $715 \ldots \ldots$ & 1.47 & 0.018 & 0.154 & 2.84 & 1.67 & 1.18 & 1.39 & 0.014 & 2.26 & 27.4 & -0.76 \\
\hline $723 \ldots \ldots$ & 1.84 & -0.025 & 0.042 & 0.90 & 0.09 & 0.04 & 0.33 & -0.000 & 1.88 & 31.0 & -2.24 \\
\hline $741 \ldots \ldots$ & 1.38 & 0.088 & 0.209 & 3.77 & 2.20 & 2.34 & 3.33 & 0.043 & 1.53 & 42.7 & -0.30 \\
\hline $746 \ldots \ldots$ & 1.77 & 0.003 & 0.155 & 2.94 & 1.98 & 1.96 & 2.44 & 0.024 & 2.63 & 78.4 & -0.57 \\
\hline $750 \ldots \ldots$ & 0.93 & 0.021 & 0.069 & 1.85 & 1.12 & 0.91 & 0.97 & 0.002 & $\ldots$ & 40.9 & -1.25 \\
\hline $770 \ldots \ldots$ & 2.19 & 0.009 & 0.136 & 2.19 & 1.51 & 1.47 & 2.37 & 0.011 & $\ldots$ & 45.5 & -0.76 \\
\hline $784 \ldots \ldots$ & 1.58 & -0.025 & 0.053 & 2.57 & 2.07 & 2.00 & 2.13 & 0.001 & 1.77 & 64.6 & -0.68 \\
\hline
\end{tabular}


TABLE 2-Continued

\begin{tabular}{|c|c|c|c|c|c|c|c|c|c|c|c|}
\hline Identity & $\begin{array}{l}\mathrm{H} \beta \\
(\AA)\end{array}$ & $\begin{array}{c}\mathrm{Mg}_{1} \\
\text { (mag) }\end{array}$ & $\underset{\text { (mag) }}{\mathrm{Mg}_{2}}$ & $\underset{(\AA)}{\operatorname{Mg} b}$ & $\begin{array}{c}\mathrm{Fe} \lambda 25270 \\
(\AA)\end{array}$ & 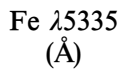 & $\begin{array}{c}\mathrm{NaD} \\
(\AA)\end{array}$ & $\begin{array}{l}\mathrm{TiO}_{1} \\
\text { (mag) }\end{array}$ & $\begin{array}{l}\mathrm{H} \alpha \\
(\AA)\end{array}$ & QSNR & $\begin{array}{c}{[\mathrm{Fe} / \mathrm{H}]_{Z}} \\
(\mathrm{dex})\end{array}$ \\
\hline $787 \ldots$ & 2.54 & -0.014 & 0.035 & 1.69 & 2.10 & 1.19 & 1.01 & 0.008 & 2.65 & 24.9 & -1.18 \\
\hline $796 \ldots \ldots$ & 2.80 & -0.065 & -0.001 & 1.22 & 1.16 & 0.57 & 0.76 & -0.004 & 1.41 & 41.4 & -1.56 \\
\hline $798 \ldots \ldots \ldots$ & $\ldots$ & -0.036 & 0.015 & 1.38 & 1.11 & 0.29 & 1.12 & -0.002 & 0.97 & 36.3 & -1.44 \\
\hline $809 \ldots \ldots$ & 2.07 & -0.033 & 0.013 & 1.34 & 1.46 & 1.67 & 0.92 & 0.015 & 1.70 & 44.5 & -1.33 \\
\hline $811 \ldots \ldots$ & 2.19 & 0.000 & 0.009 & 0.18 & -0.20 & 0.21 & -0.27 & -0.001 & 2.92 & 40.3 & -2.24 \\
\hline $824 \ldots \ldots$. & 2.11 & 0.034 & 0.110 & 2.07 & 3.94 & 1.53 & 1.76 & -0.002 & 1.95 & 32.5 & -0.80 \\
\hline $838 \ldots \ldots$. & 2.75 & -0.025 & 0.017 & 2.18 & 1.24 & 0.83 & 1.65 & 0.003 & 1.98 & 33.4 & -0.95 \\
\hline $849 \ldots \ldots$ & 2.32 & -0.006 & 0.105 & 3.73 & 2.63 & 1.83 & 2.47 & 0.038 & 2.55 & 32.8 & -0.46 \\
\hline $868 \ldots \ldots$. & 1.71 & -0.027 & 0.085 & 2.59 & 3.23 & 1.63 & 0.73 & 0.023 & 3.07 & 25.5 & -0.83 \\
\hline $871 \ldots \ldots$ & 1.31 & 0.015 & 0.113 & 3.19 & 2.89 & 2.17 & 2.75 & 0.011 & 2.41 & 27.3 & -0.49 \\
\hline $887 \ldots \ldots$. & 1.29 & -0.021 & 0.084 & 1.72 & 2.00 & 1.62 & 1.68 & 0.020 & 2.75 & 33.7 & -0.99 \\
\hline $892 \ldots \ldots \ldots$ & 1.62 & -0.016 & 0.062 & 3.24 & 1.75 & 2.97 & 2.24 & 0.032 & 2.18 & 24.6 & -0.53 \\
\hline $902 \ldots \ldots$ & 0.57 & 0.151 & 0.361 & 5.06 & 3.98 & 3.01 & 7.63 & 0.048 & 2.06 & 24.1 & +0.11 \\
\hline $910 \ldots \ldots$ & 2.72 & -0.043 & -0.042 & 0.25 & 0.47 & 1.00 & 0.66 & -0.009 & 2.58 & 39.1 & -2.24 \\
\hline $917 \ldots \ldots$ & 2.37 & -0.028 & 0.091 & 2.36 & 1.42 & 1.55 & 1.66 & 0.023 & 2.62 & 22.7 & -0.83 \\
\hline $928 \ldots \ldots$ & 1.96 & -0.032 & 0.050 & 1.32 & 1.26 & 1.08 & 1.07 & 0.005 & 2.79 & 65.0 & -1.37 \\
\hline $937 \ldots \ldots$. & 2.12 & -0.028 & 0.005 & 1.28 & 1.11 & 1.13 & 0.84 & -0.008 & 3.23 & 28.9 & -1.44 \\
\hline $941 \ldots \ldots \ldots$ & 2.11 & -0.037 & 0.020 & 0.85 & 1.26 & 0.41 & 1.54 & -0.000 & 2.54 & 46.7 & -1.59 \\
\hline $946 \ldots \ldots$. & 1.66 & 0.077 & 0.273 & 3.74 & 4.48 & 3.25 & 2.83 & 0.019 & & 29.6 & -0.30 \\
\hline $952 \ldots \ldots$. & 1.93 & -0.049 & 0.032 & 1.38 & 1.42 & 0.94 & 1.21 & 0.005 & 3.17 & 32.2 & -1.29 \\
\hline $965 \ldots \ldots$. & 2.03 & 0.066 & 0.156 & 2.87 & 1.80 & 1.44 & 2.09 & 0.029 & $\ldots$ & 52.5 & -0.65 \\
\hline $968 \ldots \ldots$. & 2.38 & -0.006 & 0.064 & 1.95 & 1.02 & 1.95 & 1.01 & 0.013 & 2.87 & 33.5 & -1.14 \\
\hline $970 \ldots \ldots$ & 2.06 & -0.040 & 0.094 & 2.50 & 2.19 & 2.42 & 1.86 & 0.035 & 2.99 & 32.5 & -0.72 \\
\hline $991 \ldots \ldots \ldots$ & 1.68 & -0.029 & 0.053 & 1.52 & 1.41 & 1.31 & 0.92 & -0.003 & 2.67 & 44.5 & -1.29 \\
\hline $992 \ldots \ldots \ldots$ & 2.82 & -0.044 & 0.057 & 2.38 & 1.01 & 2.46 & 0.90 & 0.013 & 3.96 & 25.5 & -0.95 \\
\hline $1007 \ldots \ldots$ & 1.25 & 0.032 & 0.189 & 3.20 & 1.63 & 2.57 & 2.01 & 0.028 & $\ldots$ & 58.2 & -0.57 \\
\hline $1010 \ldots \ldots$ & 0.98 & 0.049 & 0.241 & 4.37 & 2.45 & 2.15 & 3.61 & 0.043 & $\ldots$ & 38.3 & -0.19 \\
\hline $1016 \ldots \ldots$ & 1.14 & 0.159 & 0.398 & 5.65 & 3.38 & 3.42 & 7.46 & 0.037 & & 37.1 & +0.11 \\
\hline $1019 \ldots \ldots$ & 2.02 & -0.039 & 0.056 & 1.89 & 0.87 & 1.50 & 1.12 & 0.001 & 3.09 & 74.9 & -1.18 \\
\hline $1032 \ldots \ldots$ & 3.29 & -0.007 & 0.127 & 2.47 & 2.09 & 1.33 & 1.42 & 0.012 & 2.57 & 41.3 & -0.83 \\
\hline $1034 \ldots \ldots$ & 2.09 & 0.057 & 0.135 & 2.26 & 1.38 & 1.63 & 1.75 & 0.017 & & 39.3 & -0.83 \\
\hline $1044 \ldots \ldots$ & 2.24 & -0.070 & 0.015 & 2.00 & 1.96 & 0.89 & 1.39 & 0.002 & 2.94 & 44.4 & -1.02 \\
\hline $1049 \ldots \ldots$ & 1.68 & 0.015 & 0.169 & 3.87 & 3.00 & 2.20 & 2.62 & 0.019 & & 24.6 & -0.38 \\
\hline $1060 \ldots \ldots$ & 1.64 & 0.005 & 0.101 & 1.08 & 1.18 & 2.02 & 1.26 & -0.013 & 1.73 & 22.7 & -1.29 \\
\hline $1091 \ldots \ldots$ & 1.73 & -0.011 & 0.100 & 1.74 & 1.77 & 0.39 & 2.15 & -0.009 & 2.72 & 29.7 & -0.99 \\
\hline $1093 \ldots \ldots$ & & -0.004 & 0.073 & 2.21 & 2.06 & 1.44 & 1.46 & 0.016 & 0.64 & 48.5 & -0.87 \\
\hline $1101 \ldots \ldots$ & 1.75 & -0.013 & 0.127 & 1.47 & 2.60 & 1.97 & 1.99 & 0.019 & 2.28 & 28.6 & -0.95 \\
\hline $1110 \ldots \ldots$ & 2.90 & -0.062 & 0.011 & 1.45 & 1.21 & 0.88 & 1.27 & 0.001 & 3.01 & 22.1 & -1.29 \\
\hline $1113 \ldots \ldots$ & 2.16 & -0.011 & 0.044 & 0.39 & -0.08 & 0.64 & 1.11 & 0.019 & $\ldots$ & 25.9 & -2.17 \\
\hline $1116 \ldots \ldots$ & 2.51 & 0.015 & 0.147 & 2.44 & 2.56 & 0.58 & 1.67 & 0.006 & $\ldots$ & 28.5 & -0.80 \\
\hline $1119 \ldots \ldots$ & 2.12 & -0.044 & 0.109 & 1.97 & 1.40 & 1.92 & 2.06 & -0.006 & $\ldots$ & 23.5 & -0.87 \\
\hline $1120 \ldots \ldots$ & 2.29 & -0.009 & 0.090 & 1.45 & 1.72 & 1.46 & 0.80 & -0.007 & $\ldots$ & 26.0 & -1.33 \\
\hline $1155 \ldots \ldots$ & 1.28 & 0.060 & 0.240 & 4.02 & 2.55 & 2.23 & 3.26 & 0.024 & $\ldots$ & 43.0 & -0.30 \\
\hline $1157 \ldots \ldots$ & 1.45 & -0.033 & 0.022 & 1.52 & 1.84 & 1.77 & 1.22 & 0.011 & 3.18 & 46.0 & -1.14 \\
\hline $1158 \ldots \ldots$ & 1.87 & -0.008 & 0.118 & 1.31 & 0.98 & 1.01 & 1.35 & 0.005 & $\ldots$ & 33.4 & -1.33 \\
\hline $1167 \ldots \ldots$ & 2.23 & -0.041 & 0.027 & 0.99 & 0.56 & 0.57 & 0.46 & 0.001 & $\ldots$ & 23.9 & -1.86 \\
\hline $1180 \ldots \ldots$ & 1.98 & 0.013 & 0.117 & 1.73 & 1.62 & 2.11 & 1.49 & 0.020 & $\ldots$ & 65.8 & -1.02 \\
\hline $1181 \ldots \ldots$ & 0.67 & 0.020 & 0.169 & 2.99 & 1.78 & 1.73 & 1.70 & 0.012 & $\ldots$ & 27.3 & -0.68 \\
\hline $1205 \ldots \ldots$ & 2.13 & -0.060 & 0.007 & 0.90 & 0.58 & 0.79 & 1.94 & 0.006 & 2.60 & 26.1 & -1.37 \\
\hline $1238 \ldots \ldots$ & 2.36 & 0.007 & 0.085 & 2.38 & 0.98 & 1.85 & 1.10 & 0.022 & 2.60 & 28.5 & -0.95 \\
\hline $1240 \ldots \ldots$ & 1.08 & 0.004 & 0.152 & 4.39 & 3.08 & 2.69 & 2.24 & 0.024 & 2.52 & 37.2 & -0.38 \\
\hline $1247 \ldots \ldots$ & 2.39 & -0.023 & 0.072 & 2.54 & 1.60 & 1.01 & 2.54 & 0.013 & 3.07 & 37.7 & -0.68 \\
\hline $1290 \ldots \ldots$ & 2.43 & -0.016 & 0.142 & 2.60 & 2.14 & 1.04 & 1.25 & 0.007 & 2.87 & 24.3 & -0.83 \\
\hline $1293 \ldots \ldots$ & 1.67 & -0.011 & 0.051 & 2.18 & 2.27 & 1.19 & 2.11 & 0.032 & 1.59 & 32.3 & -0.80 \\
\hline $1309 \ldots \ldots$ & 1.69 & -0.007 & 0.089 & 2.79 & 2.49 & 1.83 & 2.65 & 0.018 & 2.61 & 50.1 & -0.57 \\
\hline $1336 \ldots \ldots$ & 2.46 & -0.044 & 0.091 & 1.89 & 1.65 & 1.27 & 1.50 & 0.008 & 2.80 & 50.8 & -1.02 \\
\hline $1344 \ldots \ldots$ & 2.34 & -0.018 & 0.080 & 2.84 & 1.92 & 1.23 & 2.05 & 0.018 & 2.63 & 65.3 & -0.68 \\
\hline $1351 \ldots \ldots$ & 1.70 & -0.009 & 0.089 & 3.20 & 2.12 & 1.85 & 2.00 & 0.004 & 2.06 & 53.9 & -0.61 \\
\hline $1353 \ldots \ldots$ & 2.08 & -0.033 & 0.037 & 0.60 & 1.94 & 1.65 & 0.87 & 0.002 & 3.45 & 25.5 & -1.59 \\
\hline $1370 \ldots \ldots$ & 1.91 & -0.010 & 0.131 & 3.25 & 2.51 & 1.97 & 2.62 & 0.022 & 2.50 & 83.6 & -0.49 \\
\hline $1382 \ldots \ldots$ & 2.00 & -0.062 & -0.038 & 0.98 & 0.73 & 0.42 & 0.53 & -0.002 & 2.42 & 38.0 & -1.86 \\
\hline $1431 \ldots \ldots$ & 2.54 & -0.086 & -0.070 & 1.01 & -1.53 & -0.68 & 0.86 & -0.009 & 2.90 & 29.4 & -2.24 \\
\hline $1449 \ldots \ldots$ & 2.42 & -0.056 & -0.017 & 1.67 & 1.26 & 0.15 & 1.03 & 0.014 & 1.52 & 31.8 & -1.37 \\
\hline $1463 \ldots \ldots$ & 1.42 & -0.075 & 0.002 & 0.87 & 1.59 & 1.43 & 1.71 & 0.015 & 2.45 & 36.3 & -1.25 \\
\hline $1479 \ldots \ldots$ & 1.96 & -0.029 & 0.071 & 1.59 & 1.20 & 1.73 & 0.82 & 0.011 & 2.93 & 41.8 & -1.29 \\
\hline $1481 \ldots \ldots$ & 2.03 & -0.031 & 0.048 & 1.58 & 1.59 & 1.28 & 2.00 & 0.007 & 2.83 & 38.3 & -1.02 \\
\hline $1483 \ldots \ldots$ & 2.65 & -0.096 & -0.016 & 0.54 & 0.64 & 2.21 & 1.39 & 0.010 & 2.11 & 23.2 & -1.63 \\
\hline $1504 \ldots \ldots$ & 2.09 & -0.068 & -0.061 & 0.52 & 0.29 & 0.51 & 0.54 & 0.018 & 1.88 & 52.7 & -2.24 \\
\hline $1514 \ldots \ldots$ & 1.86 & 0.036 & 0.166 & 4.54 & 2.55 & 2.61 & 2.83 & 0.028 & 1.30 & 48.2 & -0.27 \\
\hline $1538 \ldots \ldots$ & 1.63 & 0.004 & 0.094 & 2.86 & 2.46 & 2.19 & 2.08 & 0.032 & 1.83 & 70.1 & -0.61 \\
\hline $1540 \ldots \ldots$ & 2.05 & 0.023 & 0.170 & 3.63 & 2.86 & 2.24 & 1.94 & 0.014 & 2.31 & 28.1 & -0.49 \\
\hline $1548 \ldots \ldots$ & 2.39 & 0.011 & 0.144 & 2.36 & 2.53 & 2.10 & 1.20 & 0.015 & 2.02 & 61.6 & -0.83 \\
\hline
\end{tabular}


TABLE 2-Continued

\begin{tabular}{|c|c|c|c|c|c|c|c|c|c|c|c|}
\hline Identity & $\begin{array}{l}\mathrm{H} \beta \\
(\AA)\end{array}$ & $\begin{array}{c}\mathrm{Mg}_{1} \\
(\mathrm{mag})\end{array}$ & $\begin{array}{c}\mathrm{Mg}_{2} \\
(\mathrm{mag})\end{array}$ & $\underset{(\AA)}{\operatorname{Mg} b}$ & $\begin{array}{c}\mathrm{Fe} \lambda 5270 \\
(\AA)\end{array}$ & 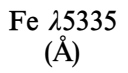 & $\begin{array}{c}\mathrm{NaD} \\
(\AA)\end{array}$ & $\begin{array}{l}\mathrm{TiO}_{1} \\
\text { (mag) }\end{array}$ & $\begin{array}{l}\mathrm{H} \alpha \\
(\AA)\end{array}$ & $Q S N R$ & $\begin{array}{c}{[\mathrm{Fe} / \mathrm{H}]_{Z}} \\
(\mathrm{dex})\end{array}$ \\
\hline 1563. & 2.60 & -0.033 & 0.057 & 2.28 & 2.35 & 0.72 & 0.97 & -0.005 & 2.91 & 25.6 & -0.99 \\
\hline $1565 \ldots \ldots$ & 2.60 & -0.057 & 0.030 & 1.26 & 1.58 & 1.26 & 0.70 & 0.003 & 3.15 & 34.4 & -1.44 \\
\hline $1594 \ldots \ldots$ & 2.09 & -0.021 & 0.058 & 1.73 & 1.68 & 1.33 & 1.34 & 0.021 & 2.42 & 37.1 & -1.10 \\
\hline $1615 \ldots \ldots$ & 2.13 & -0.020 & 0.049 & 2.26 & 1.61 & 1.67 & 1.22 & 0.012 & 2.67 & 55.8 & -0.95 \\
\hline $1617 \ldots \ldots$ & 1.61 & -0.032 & 0.054 & 2.18 & 1.97 & 1.46 & 1.21 & 0.004 & 2.99 & 74.6 & -0.95 \\
\hline $1631 \ldots \ldots$ & 1.54 & -0.029 & 0.049 & 1.14 & 2.61 & 1.26 & 1.30 & 0.004 & 3.23 & 38.4 & -1.22 \\
\hline $1664 \ldots \ldots$ & 1.58 & -0.030 & 0.085 & 2.58 & 1.96 & 0.66 & 1.81 & 0.026 & 2.82 & 51.6 & -0.76 \\
\hline $1709 \ldots \ldots$ & 1.22 & 0.015 & 0.177 & 2.75 & 2.14 & 2.46 & 1.68 & 0.014 & 2.73 & 22.9 & -0.68 \\
\hline
\end{tabular}

Each spectrum was checked to make sure there would be no problems with either of the Balmer line indices due to a bandpass slipping beyond the range of the valid data.

\subsection{Measured Indices}

Table 2 contains the final list of measured indices for the M87 GCs together with their $Q S N R$ values.

The errors for the indices have been calculated assuming Poisson statistics, taking into account the number of pixels averaged to form the numerator and denominator of the index, and also the change in mean signal level (in data numbers) of the spectra as a function of wavelength. This last is a factor of 2.5 from the lowest mean signal level in data numbers at the blue end of the $\mathrm{H} \beta$ index to the higher signal levels prevailing at the redder indices. Assuming $Q S N R=22$ predicts errors of $0.5 \AA$ for the $\mathrm{H} \beta$ index, the same for the $\mathrm{Mg} b$ index, and $0.3 \AA$ for the $\mathrm{H} \alpha$ index. The predicted error for the $\mathrm{TiO}_{1}$ index is smaller, as many more pixels are averaged in each of its bandpasses. These calculated values do not take into account cosmic rays or the systematic problems of matching the global continuum slope discussed above. Also the above were predicated on the smooth base level of the night sky and do not include the individual strong resolved features such as the $\mathrm{NaD}$ lines that fall within the redder indices.

The mean of the absolute value of the differences in the measured indices for the 12 M87 GCs with multiple spectra is $0.5 \AA$ or less for the five indices we use. This ignores three outliers among the 60 cases.

A foreground extinction of $E(B-V)=0.022 \mathrm{mag}$ to M87 (Burstein \& Heiles 1984) and the reddening law of Reed, Hesser, \& Shawl (1988) are adopted for the M87 GCs.

\section{CALIBRATION VIA THE GALACTIC GLOBULAR CLUSTERS}

As a calibration for the M87 globular clusters, we rely on observations of galactic globular clusters. We have scanned the cores of 12 galactic globular clusters covering a wide range in metallicity using the same instrumental configuration as was used for our M87 GC observations. The scans are typically $90^{\prime \prime}$ in length and we use a $3^{\prime}$ long slit, so the stellar population within a cluster is reasonably well sampled. Each scan was repeated several times, and scans in background fields well offset from the cluster were sometimes acquired as well. In the reduction of the scans, very bright isolated stars were cut out of the part of the image used. An effort was made to include several very metal-rich galactic globular clusters in this group.

The very metal-rich globular clusters are usually seen against the strong background of the galactic bulge, and proper sky subtraction, particularly for the most diffuse or faintest of them, becomes an issue. We have tried various schemes for sky subtraction and can alter the measured $\mathrm{H} \beta$ by not more than $10 \%$ and the other indices by even less. NGC 6539 is the most difficult case among the ones we observed.

The measured indices in the Lick system for the galactic globular clusters from our Keck + LRIS data are listed in Table 3. We supplement these data by the observations of Burstein et al. (1984). Six of our sample of galactic GCs overlap their sample, and the intercomparison of the indices is very encouraging.

We adopt the abundances of Zinn (1985) for the galactic globular clusters. The $U B V R I$ colors for the galactic globular clusters are taken from the compilation of Harris (1996). Several of the galactic globular clusters lack photometry at $R$, and their $(U-R)$ colors were obtained by interpolating for $(V-R)$ from mean relationships for the galactic globulars given by Reed et al. (1988) combined with their observed $U B V$ colors. The extinction ratios of Reed et al. (1988) are adopted, specifically $E(U-R) / E(B-V)=2.54$. There are two galactic GCs in our sample with $E(B-V)>0.8$, NGC $6539(E[B-V]=1.00)$ and NGC

TABLE 3

Index Measurements for Galactic Globular Clusters

\begin{tabular}{ccccccccccc}
\hline \hline $\begin{array}{c}\text { Globular } \\
\text { Cluster }\end{array}$ & $\begin{array}{c}\mathrm{H} \beta \\
(\AA)\end{array}$ & $\begin{array}{c}\mathrm{Mg}_{1} \\
(\mathrm{mag})\end{array}$ & $\begin{array}{c}\mathrm{Mg}_{2} \\
(\mathrm{mag})\end{array}$ & $\begin{array}{c}\mathrm{Mg} b \\
(\AA)\end{array}$ & $\begin{array}{c}\mathrm{Fe} \lambda 5270 \\
(\AA)\end{array}$ & $\begin{array}{c}\mathrm{Fe} \lambda 5335 \\
(\AA)\end{array}$ & $\begin{array}{c}\mathrm{NaD} \\
(\AA)\end{array}$ & $\begin{array}{c}\mathrm{TiO}_{1} \\
(\mathrm{mag})\end{array}$ & $\begin{array}{c}\mathrm{H} \alpha \\
(\AA)\end{array}$ & $\begin{array}{c}{[\mathrm{Fe} / \mathrm{H}]_{Z} \pm} \\
(\mathrm{dex})\end{array}$ \\
\hline $\mathrm{M} 92 \ldots \ldots \ldots$ & 2.53 & 0.002 & 0.028 & 0.57 & 0.53 & 0.37 & 0.67 & 0.001 & 2.35 & -2.240 .08 \\
$\mathrm{M} 13 \ldots \ldots \ldots$ & 2.40 & 0.007 & 0.048 & 0.95 & 0.92 & 0.71 & 0.90 & 0.002 & 2.29 & -1.650 .06 \\
$\mathrm{M} 4 \ldots \ldots \ldots$. & 2.82 & 0.030 & 0.095 & 1.59 & 1.28 & 0.97 & 1.67 & 0.009 & 2.54 & -1.280 .10 \\
$\mathrm{~N} 6171 \ldots \ldots$. & 2.00 & 0.041 & 0.119 & 1.69 & 1.60 & 1.47 & 1.80 & 0.016 & 2.90 & -0.990 .06 \\
$\mathrm{~N} 6539 \ldots \ldots$. & 1.39 & 0.085 & 0.195 & 2.86 & 2.30 & 1.77 & 3.76 & 0.043 & 1.52 & -0.660 .15 \\
$\mathrm{~N} 6356 \ldots \ldots$. & 1.62 & 0.070 & 0.169 & 3.09 & 2.00 & 1.69 & 3.00 & 0.029 & $\ldots$ & -0.620 .20 \\
$\mathrm{M} 71 \ldots \ldots$. & 1.29 & 0.071 & 0.158 & 2.84 & 1.80 & 1.64 & 2.25 & 0.022 & $\ldots$ & -0.580 .08 \\
$\mathrm{~N} 6760 \ldots \ldots$ & 1.34 & 0.096 & 0.228 & 3.44 & 2.52 & 2.01 & 3.08 & 0.050 & 1.26 & -0.520 .15 \\
$\mathrm{~N} 6624 \ldots \ldots$. & 1.69 & 0.048 & 0.163 & 2.94 & 2.09 & 1.78 & 2.20 & 0.035 & 1.63 & -0.350 .15 \\
$\mathrm{~N} 6553 \ldots \ldots$. & 1.63 & 0.110 & 0.249 & 3.88 & 3.11 & 2.51 & 3.40 & 0.044 & 1.26 & -0.290 .11 \\
$\mathrm{~N} 6440 \ldots \ldots$ & 1.34 & 0.103 & 0.227 & 3.31 & 2.50 & 1.96 & 3.94 & 0.034 & 1.48 & -0.260 .15 \\
$\mathrm{~N} 6528 \ldots \ldots$. & 1.80 & 0.097 & 0.247 & 3.89 & 2.96 & 2.45 & 4.93 & 0.046 & 1.29 & +0.120 .21 \\
\hline
\end{tabular}


$6440(E[B-V]=1.09)$. In those two cases, $(U-R)_{0}$ is quite uncertain.

It should be noted that these scans cover only the cores/ central regions of the galactic GCs, while our spectra of the M87 GCs include essentially all the integrated light of each cluster. If there is strong mass segregation in galactic GCs, differential shifts in the colors and indices of the galactic versus the M87 GCs could be produced. This does not appear to be the case.

\section{QUALITATIVE ANALYSIS}

We present first an analysis that is free of any models (and of the uncertainty of any models). Hence it is qualitative in nature, comparing the M87 GCs with the galactic GCs. We began by plotting various indices as a function of $(U-R)$ color, adopting the optical photometry of Strom et al. (1981) for the M87 GCs. Suitable photometry is not given in Strom et al. (1981) for some of the clusters in his sample and is also not available for the objects close to the nucleus of M87 that were added in Paper I. In some cases we measured $(U-R)$ (see Appendix B), but for four clusters close to the M87 nucleus no photometry is available, and they are omitted from the figures where $(U-R)$ is used (Figs. 2-8, 18, and 19). These four M87 GCs are not uniformly at the extreme in any parameter, and omitting them does not noticeably modify any of the trends displayed in these figures.

Each of the figures in this section has two panels. In the first (top), the indices of each of the M87 GCs are shown. Large, filled circles represent objects with $Q S N R \geq 40$. The $1 \sigma$ error bars shown are those appropriate to an object with the minimum $Q S N R$ to be included in the sample. In the second (bottom) panel of each figure the galactic globular clusters from our sample are shown as asterisks, while those from Burstein et al. (1984) are shown as open circles. The measurements for those clusters in common in the two galactic GC samples are connected by straight lines. Here the M87 data is represented as a solid line, the "median line" defined by Press et al. (1986), which minimizes the total absolute deviation of the points. The dashed lines are lines with the same slope as the median line but that enclose $75 \%$ of the points on each side of the median line. Thus the two dashed lines enclose $75 \%$ of the distribution of the M87 GCs.

Inspection of the scans of the galactic GCs shows that the most metal-rich among them have about $15 \%$ absorption in the $6250 \AA$ band of TiO. The absorption in the $5950 \AA$ band of $\mathrm{TiO}$ that is measured by the $\mathrm{TiO}_{1}$ index of the Lick system, while still visible in the scans, is about $\frac{1}{3}$ as large. We thus expect the $\mathrm{TiO}_{1}$ index to range from 0 in the more metal-poor galactic (and M87) GCs to about 5\% absorption in the most metal-rich ones.

This behavior is in fact shown in Figure 2, which presents the $\mathrm{TiO}_{1}$ index as a function of $(U-R)$ color. We use Figure 2 to verify that any difference in global continuum slope between our measurements and the original Lick IDS spectra is not introducing noticeable errors. The $\mathrm{TiO}_{1}$ index encompasses a range of $285 \AA$ (Table 1), far more than any other index we use. Note that this index is expressed as a magnitude difference, unlike all the others we use, which are expressed as equivalent widths (EW in Table 1) of absorption.

The $\mathrm{TiO}_{1}$ index becomes more positive as the object becomes redder, and the total range over the full range of

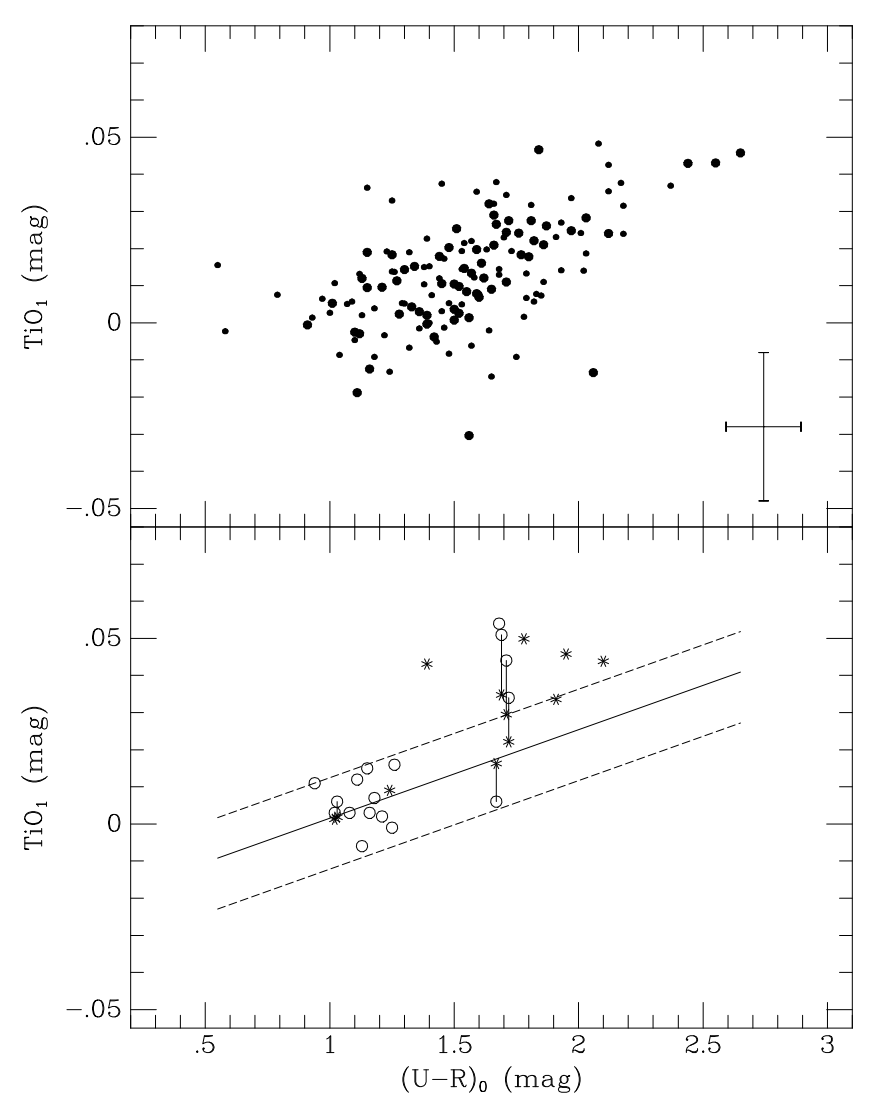

FIG. 2.- $\mathrm{TiO}_{1}$ index is shown as a function of $(U-R)$ color. The upper panel shows the data for the M87 GCs, with objects with $Q S N R \geq 40$ indicated by the larger circles. In the lower panel the data for the galactic GCs (asterisks, our data; open circles, Burstein et al. 1984) is compared with the median line of the M87 sample.

the data set is that predicted above. More importantly, the comparison between the Burstein et al. (1984) indices from Lick observations of galactic globular clusters and our indices indicates a difference of not more than $2 \%$ in this index, with perhaps a small mean difference in slope. Given that this is the broadest index, we feel that continuum slope differences should not be a serious problem for the other indices we measure. However a $2 \%$ error in continuum placement for an index $30 \AA$ wide corresponds to a $0.6 \AA$ error in equivalent width, and the range in strength of absorption from very metal-poor to very metal-rich in the $\mathrm{Mg} b$ index for example is only $6 \AA$, so continuum errors must be watched carefully.

Figure 3 shows $\mathrm{Mg} b$ versus the color $(U-R)$. As expected $\mathrm{Mg} b$ becomes stronger in the redder M87 GCs, and the scatter is consistent with there being a single monotonic relationship between them. The lower panel shows that the median line followed by the M87 GCs is also followed by the galactic globular clusters. Figure 4 shows the $\mathrm{Fe} \lambda 5270$ blend index versus $(U-R)$, and Figure 5 shows the $\mathrm{Fe} \lambda 5335$ blend index versus $(U-R)$. While the total range in these two indices for the M87 GC sample is not as large as that of $\mathrm{Mg} b$, in both cases again the index becomes stronger as the color becomes redder. In both cases the galactic globular clusters follow the median line of the M87 GCs quite closely.

The NaD line index as a function of $(U-R)$ color is shown in Figure 6. There are six M87 GCs that have extremely strong $\mathrm{NaD}$ lines $(\mathrm{EW}>4.5 \AA)$. One of them 


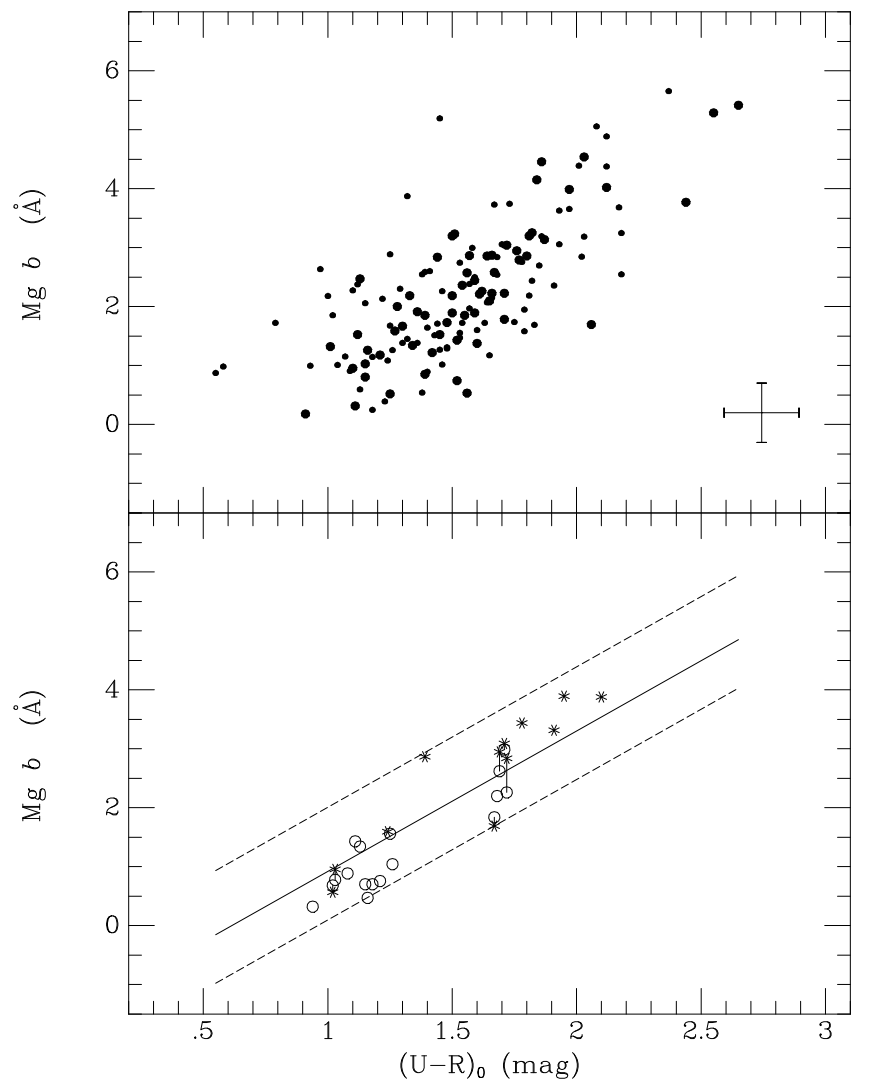

FIG. 3.-The same as Fig. 2 but for the $\mathrm{Mg} b$ index

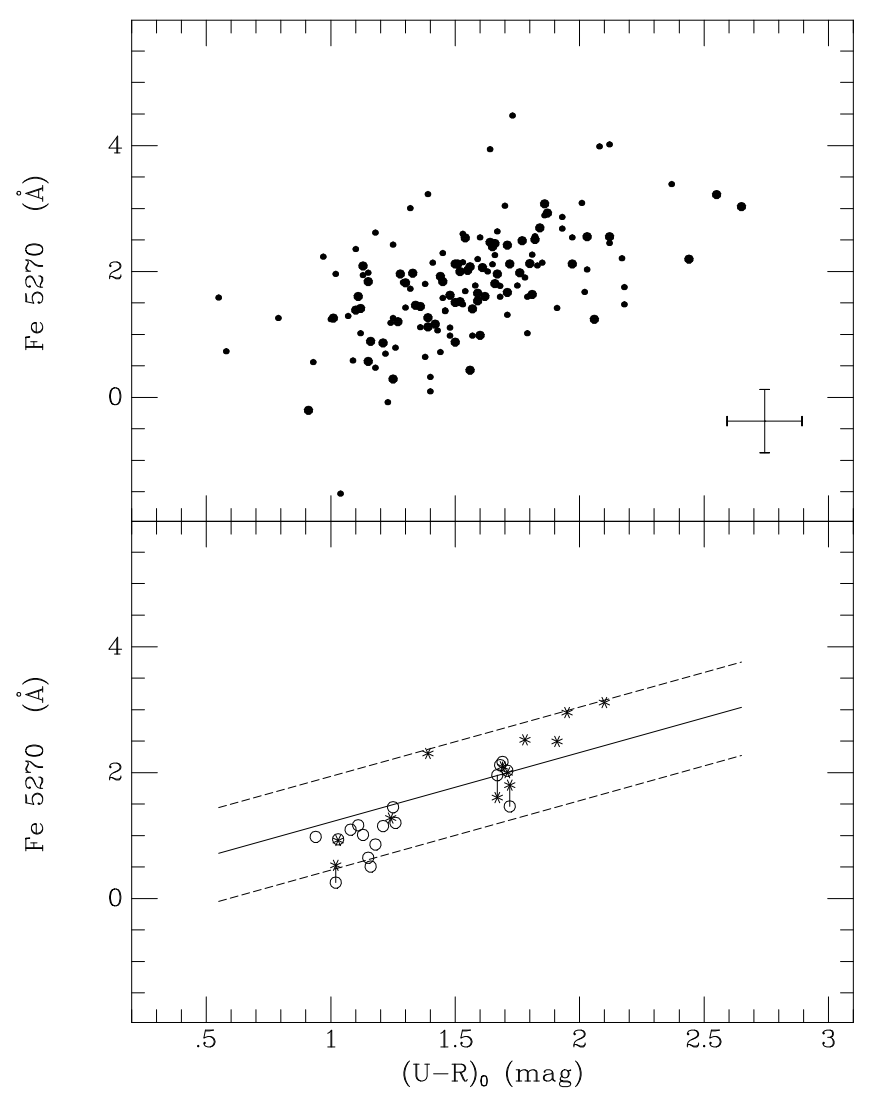

FIG. 4.-The same as Fig. 2 but for the Fe $\lambda 5270$ index

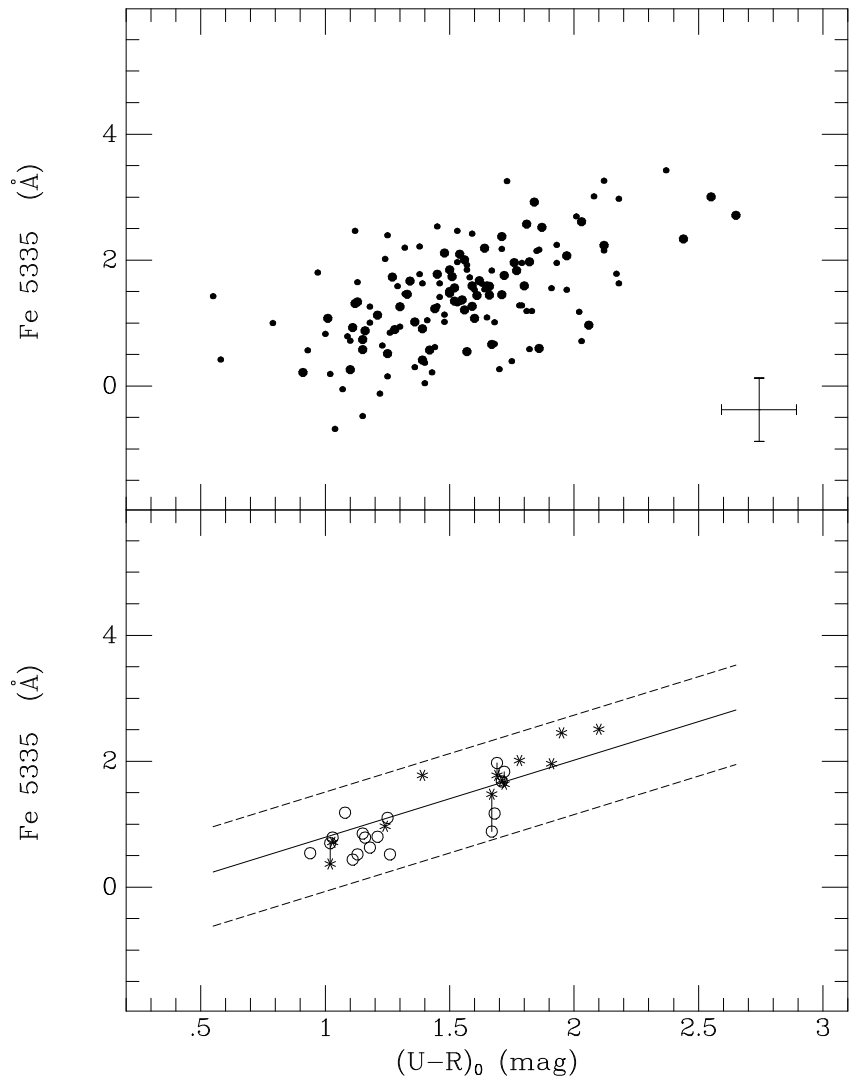

Fig. 5.-The same as Fig. 2 but for the Fe $\lambda 5335$ index

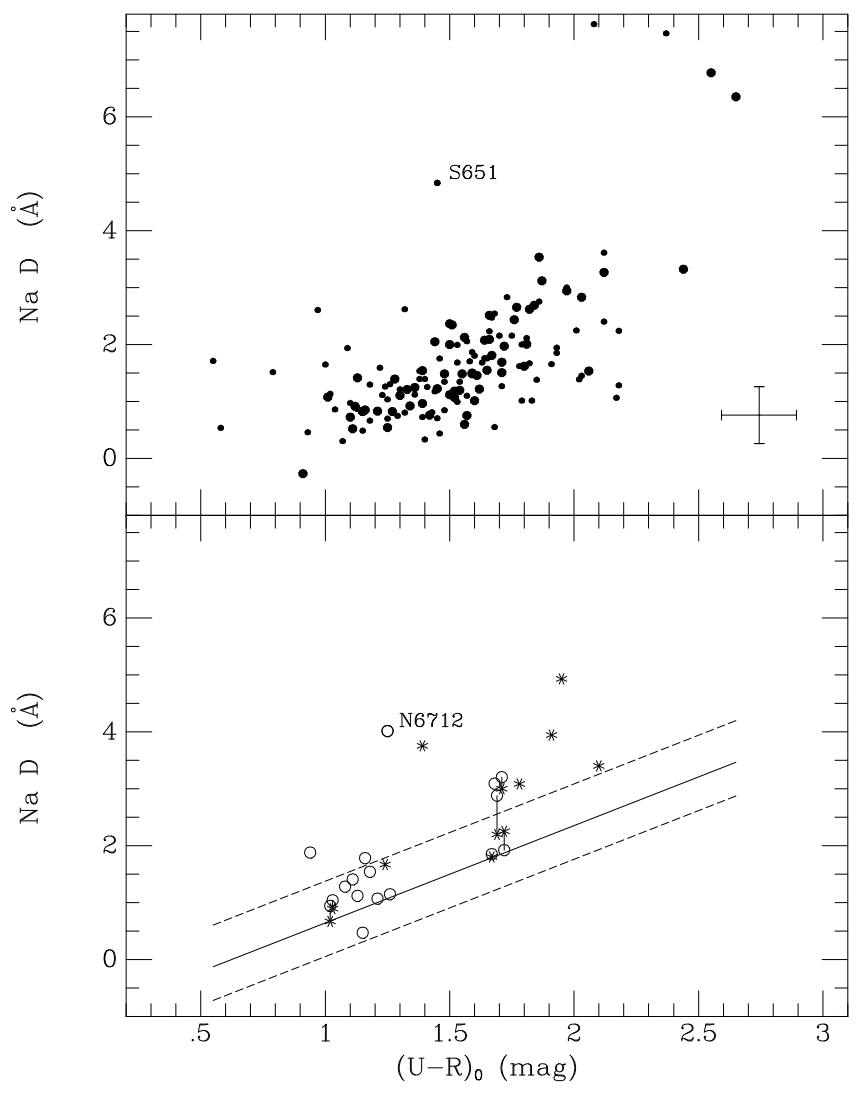

Fig. 6.-The same as Fig. 2 but for the $\mathrm{NaD}$ index 
lacks a color measurement and another, Strom 651, is discussed below; the rest are very red in $(U-R)$. Also, the galactic clusters are in the mean displaced somewhat above the median line of the M87 GCs. The object that has the largest vertical displacement is NGC 6712. In this case it is clear that the measured $\mathrm{NaD}$ index is being influenced by strong interstellar lines. At galactic coordinates of $\left(25^{\circ}\right.$, $-4^{\circ}$ ), and with a distance of $\approx 7 \mathrm{kpc}$, there is ample opportunity for substantial interstellar absorption to occur along the line of sight to this moderately metal-poor galactic globular cluster. The very metal-rich galactic GCs also lie above the median line, implying strong interstellar $\mathrm{NaD}$ absorption. For objects of reddening $E(B-V) \approx 1 \mathrm{mag}$ in the galactic plane (which is characteristic of the most heavily reddened of the galactic GCs), one may expect interstellar absorption in the NaD lines of $\approx 1.5 \AA$ (Hobbs 1974; Cohen 1975).

The M87 GC Strom 651 is also identified on this figure. A comparison of the $\mathrm{NaD}$ index versus $\mathrm{Mg} b$ index plot (Fig. 9) with Figure 6 suggests that this M87 GC has either been misidentified by us or has an incorrect $(U-R)$ color in Table 3 of Strom et al. (1981). We have checked, beginning with this table, the identification of Strom 651 on our charts and have checked that the correct object was in fact observed by superposing the images of the mask and of the field in M87 taken to align the slitmask. The $R$ mag of the object we observed (see Appendix B) is in good agreement with that given by Strom et al. (1981).

The Balmer line indices $\mathrm{H} \beta$ and $\mathrm{H} \alpha$ are shown in Figures 7 and 8 as a function of $(U-R)$ color. Since the $\mathrm{H} \alpha$ index was defined by us, there are no published measurements of $\mathrm{H} \alpha$ in the Lick system, so the number of galactic globular

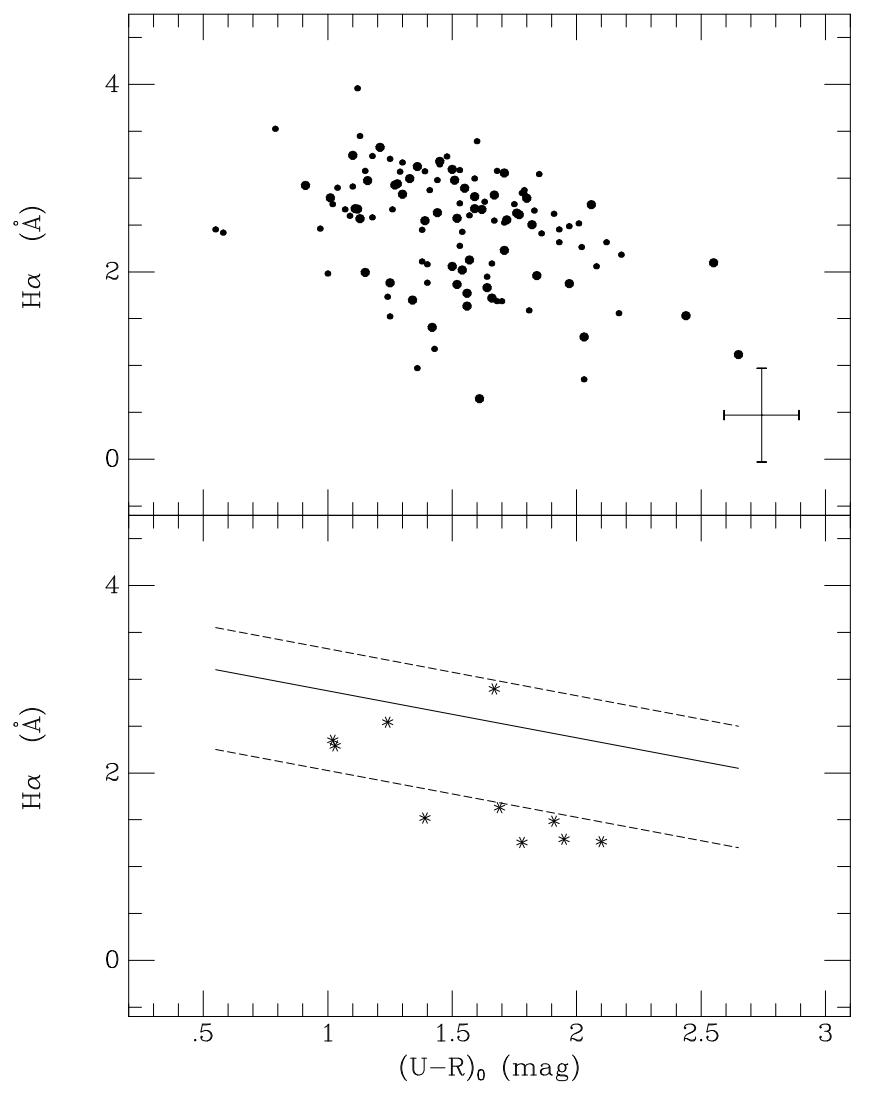

Fig. 7.- The same as Fig. 2 but for the $\mathrm{H} \alpha$ index

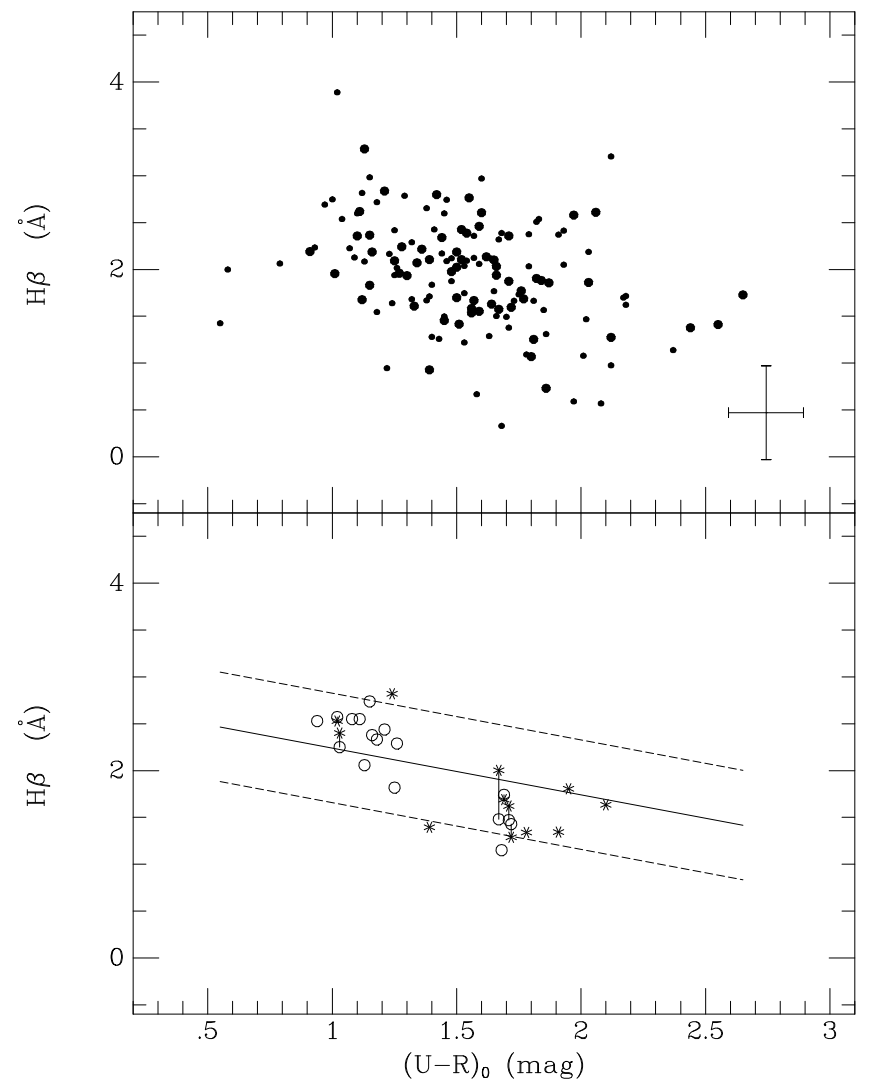

FIG. 8.-The same as Fig. 2 but for the $\mathrm{H} \beta$ index

clusters with measured indices is small. The index we have defined for $\mathrm{H} \alpha$ shows similar behavior to that of $\mathrm{H} \beta$, with the Balmer lines increasing in strength for the bluer M87 GCs, the opposite of the behavior of the metal line indices. The $\mathrm{S} / \mathrm{N}$ of the spectra is somewhat higher at $\mathrm{H} \alpha$ than at $\mathrm{H} \beta$, and this is reflected in the relative size of the dispersions in the two plots.

We now display a few index-index plots, avoiding the use of $(U-R)$ colors. This means that all 150 of the M87 GCs can now be displayed. Figure 9 shows the $\mathrm{NaD}$ line index versus the $\mathrm{Mg} b$ index. Again the galactic globulars are displaced upward from the median line of the M87 GCs. NGC 6712, where we are certain that interstellar NaD lines are contributing to the apparent strength of the $\mathrm{NaD}$ index, has the largest displacement. Strom 651 now fits onto the main M87 GC relationship, whereas it was quite anomalous in Figures 3 and 6, suggesting an error in its $(U-R)$ color.

Figure 10 shows the $\mathrm{H} \beta$ index versus $\mathrm{Mg} b$ index for the M87 GCs. As expected, stronger Mg lines are correlated with weaker Balmer lines. The Fe $\lambda 5270$ blend index is shown as a function of the $\mathrm{Mg} b$ index for the M87 and galactic GCs in Figure 11.

With the exception of the $\mathrm{NaD}$ index, where interstellar lines are believed to contaminate the galactic GC spectra, there is better agreement between our galactic globular sample and the Burstein et al. (1984) sample in Figures 3, 4, $5,7,8,10$, and 11 than occurred for the $\mathrm{TiO}_{1}$ index of Figure 2. This is also true of agreement between the M87 GC median line and the galactic globulars in the same set of figures. We consider this an indication that continuum slope problems do not affect any index at a level exceeding 


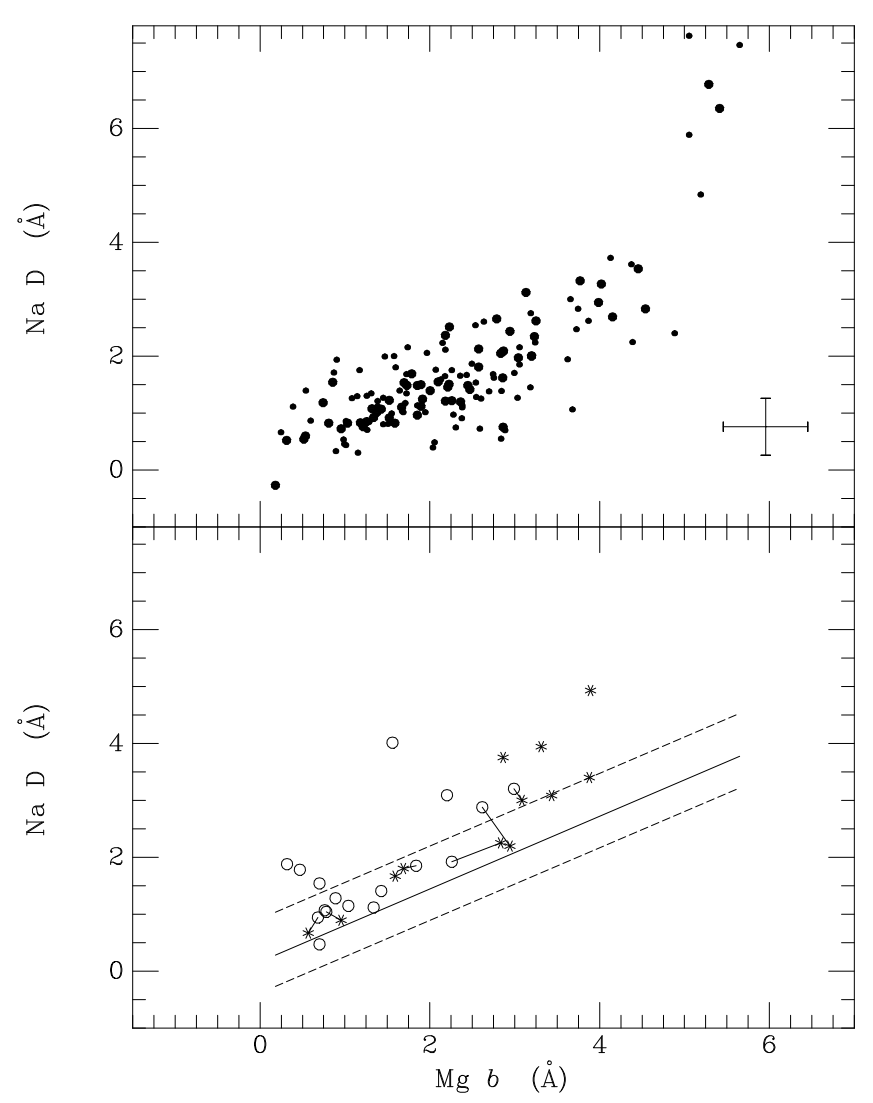

Fig. 9. $-\mathrm{NaD}$ index is shown as a function of the $\mathrm{Mg} b$ index. The upper panel shows the data for the M87 GCs, with objects with $Q S N R \geq 40$ indicated by the larger circles. In the lower panel the data for the galactic GCs (asterisks, our data; open circles, Burstein et al. 1984) is compared with the median line of the M87 sample.

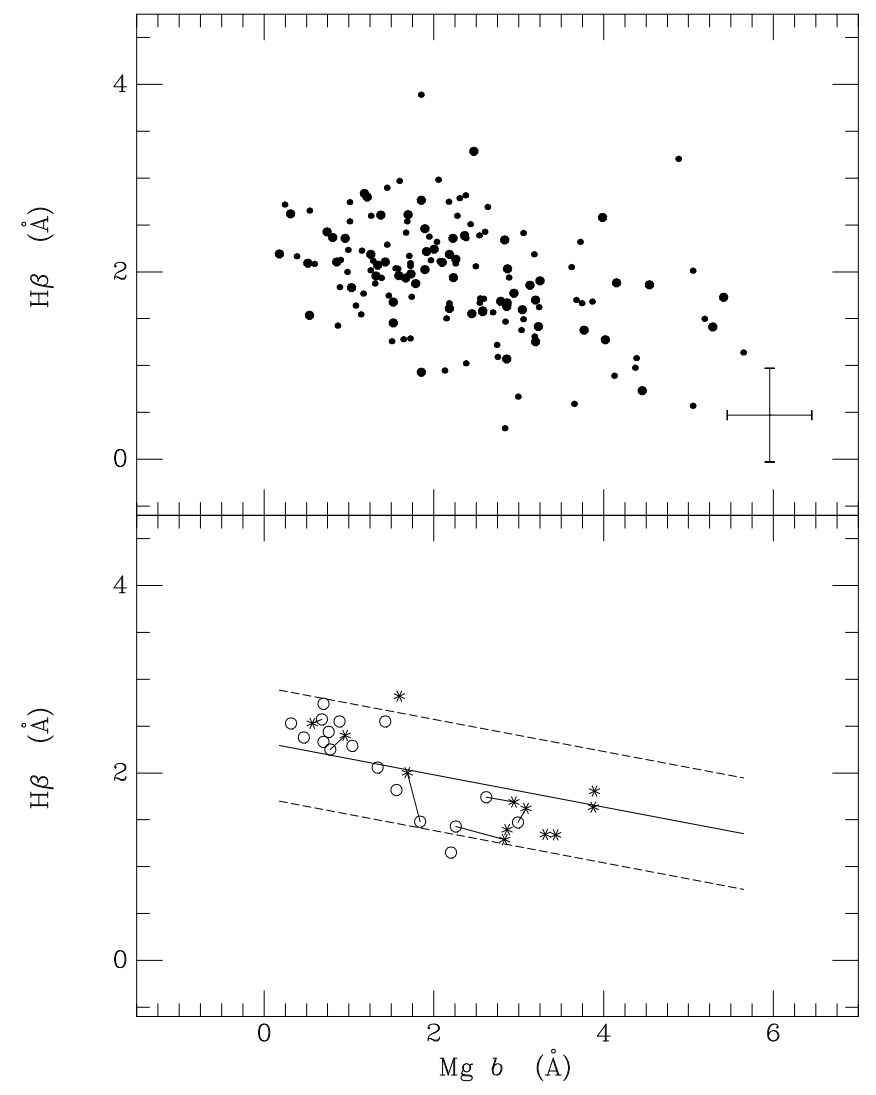

FIG. 10.-Same as Fig. 9 for the $\mathrm{H} \beta$ index

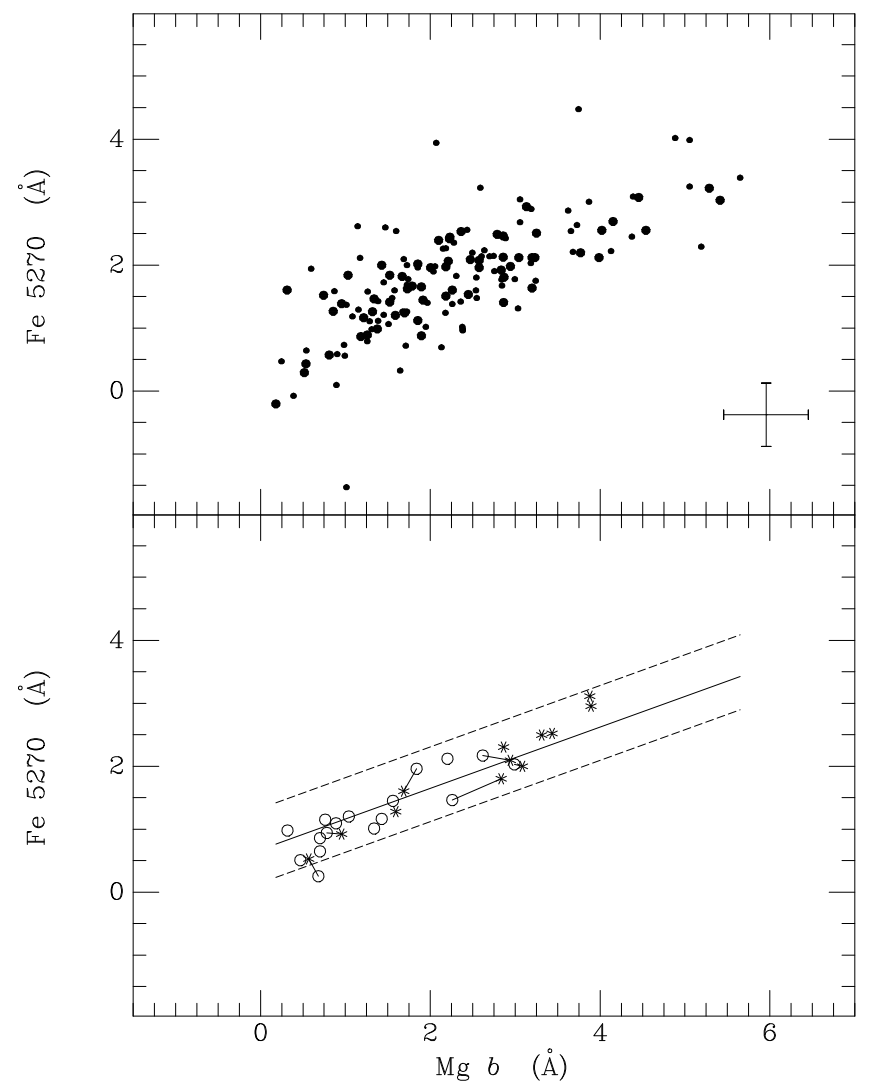

FIG. 11.-Same as Fig. 9 for the Fe $\lambda 5270$ index

$2 \%$, and perhaps affect the index with the broadest wavelength coverage, $\mathrm{TiO}_{1}$, at the $2 \%$ level.

\subsection{Qualitative Results}

The following qualitative results emerge from studying these figures:

1. The metallicity range spanned by the M87 GC system is similar to that of the galactic globulars, but the M87 GCs have a metal-rich tail that appears to extend to significantly higher metallicities than one finds among the galactic GCs. The low-metallicity end appears to be similar in the two systems, but it must be recalled that the $\mathrm{Mg} b$ index (and the absorption in the $\mathrm{Mg}$ triplet) is heading rapidly toward zero, and all discrimination will be lost at that point. It does appear, however, that in the M87 GCs the low end of the $\mathrm{Mg} b$ distribution is cut off at a finite $\mathrm{Mg} b$ index, and hence loss of discrimination is not serious.

2. The behavior of the $\mathrm{NaD}$ index in the galactic GCs as compared to the M87 sample shows evidence for a contribution to the galactic GC NaD indices by interstellar lines. Since there is not a large quantity of $10,000 \mathrm{~K}$ interstellar gas in the halo of M87, we do not expect substantial interstellar $\mathrm{NaD}$ lines to occur in the spectra of the M87 GCs.One might wonder if the few (six) M87 GCs with very large $\mathrm{NaD}$ indices also suffer interstellar absorption from gas within M87. These objects all have $R \leq 200^{\prime \prime}$ from the center of M87 but are not confined closely to the nucleus of M87, where one might expect any gas to be concentrated. Furthermore they are among the most metal-rich objects in all indices, not just in the NaD index. We hypothesize that the behavior of the $\mathrm{NaD}$ index just happens to be more 
extreme (i.e., more nonlinear) at very high metallicities than the other indices. That may be a function of the saturation of the lines, the detailed behavior of the curve of growth, etc.

3. The relative abundances of $\mathrm{Fe}, \mathrm{Na}$, and $\mathrm{Mg}$ appear to be changing together, in the same way as in the galactic globulars and in galactic stars. There are essentially no objects with extremely strong $\mathrm{Fe}$ lines but weak $\mathrm{Mg}$ lines, etc. In other words, to first order the nucleosynthesis history appears to have been the same in the M87 GC system as in the Milky Way GC system.

4. The Balmer line indices also behave similarly in the M87 and in the galactic globular clusters. It is well known that the Balmer lines are the most readily detectable agesensitive features in the integrated light spectra of old stellar systems (Rabin 1982; Worthey 1994). This similarity implies that the mean age and the age distribution are similar in these two systems.

\subsection{Radial Gradients and Trends with Luminosity}

Before proceeding to a more quantitative treatment, we address several other issues in a qualitative way.

Possible radial gradients are explored in Figure 12, which displays three of the indices as a function of projected radius $R$. The large scatter at all radii in the metallicity of the GCs in M87 makes the detection of a radial trend difficult. There is no trend in the $\mathrm{H} \beta$ index with $R$, which effectively translates into no trend of GC age with $R$. There is a small radial trend in $\mathrm{Mg} b$ index, which corresponds to a radial gradient of $\approx 0.2$ dex over the range $30^{\prime \prime} \leq R \leq 500^{\prime \prime}$. The radial gradient inferred from the NaD index is about twice as large,

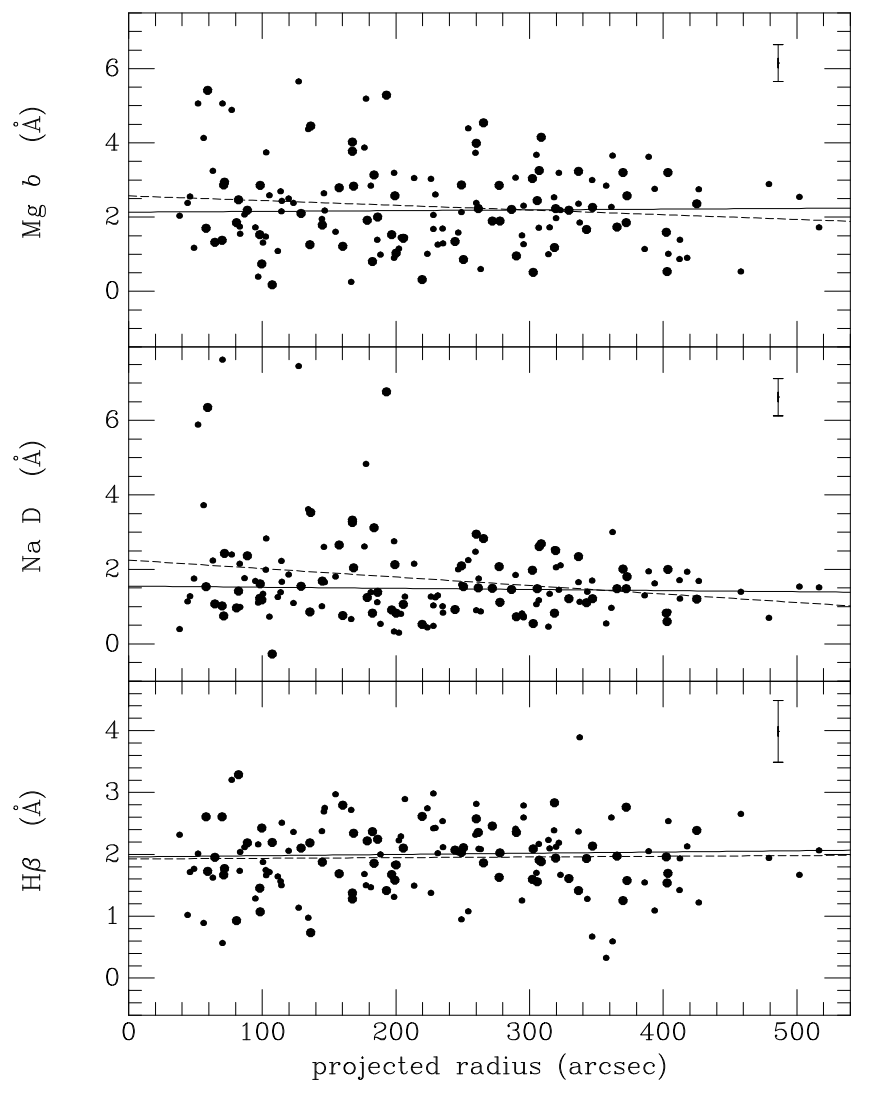

FIG. 12.- $\mathrm{Mg} b, \mathrm{NaD}$, and $\mathrm{H} \beta$ indices for the M87 GCs are shown as a function of projected radius. The least-squares fit for each index is indicated by a dashed line, while the median line is shown as a solid line. but the apparent size of the radial gradient in the $\mathrm{NaD}$ index is influenced by the six M87 GCs with extremely high $\mathrm{NaD}$ indices. The gradient for a least-squares fit is in this case significantly larger than for the median line fit. However, at all values of $R$ the scatter is much larger than any gradient. Since the observed radial gradients in the line indices are small, no effort was made to deproject them.

Although our sample of M87 GCs was selected from the magnitude-limited photometric survey of Strom et al. (1981), there are a number of effects that lead to the mean brightness of the objects in our sample becoming progressively fainter at larger $R$. The most important of these is the rapid decrease in the areal density of M87 GCs as $R$ increases. This means in practice that in designing slit masks, one will run out of bright objects in the outer part of the M87 halo long before one does so in the inner part of the halo. A second, less important, factor has to do with how the sample was defined. Our selection criterion is based not on brightness, but on QSNR, which in turn depends also on the brightness of the background. Hence a GC must be brighter to make it into the sample when the background from the M87 halo becomes brighter, i.e., at smaller $R$.

We have established that there is a radial gradient in metallicity in the M87 GC system of the expected sign (more metal-rich at smaller $R$ ), and hence this, coupled with a correlation of mean brightness with projected radius, will introduce a spurious trend of increasing metallicity with increasing brightness. This is in fact seen in our data. Although it is possible that the most massive GCs may have been able to self-enrich to some extent, increasing their mean metallicity, no analysis of a magnitude limited photometric sample has ever detected any correlation of mean color with brightness. Thus, we assume this is a spurious result introduced by the biases in our sample selection.

The objects with high QSNR are among the brighter M87 GCs, as we would expect.

\subsection{The Histogram of $\mathrm{Mg} b$ Indices for the M87 Globular Cluster System}

The histogram of $\mathrm{Mg} b$ indices (Fig. 13) has a broad peak with a metal-rich tail. While there is in principle little discrimination at extremely low metallicities, there are few very metal-poor clusters in the M87 GC system. The $1 \sigma$ error in the $\mathrm{Mg} b$ index is $0.5 \AA$ for a M87 GC with QSNR

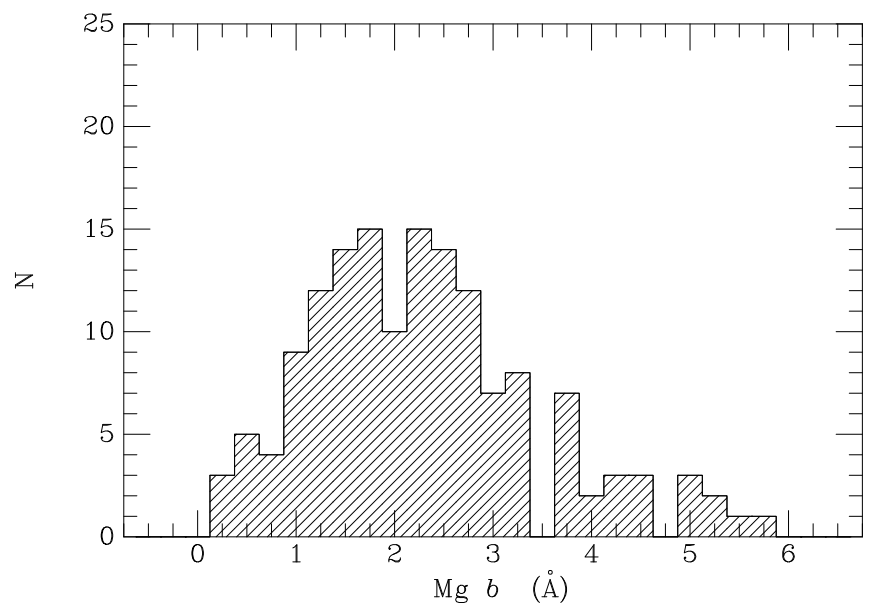

FIG. 13.- Histogram of the $\mathrm{Mg} b$ indices for $150 \mathrm{M} 87 \mathrm{GCs}$ is shown 
at the minimum required to get into the abundance sample. (The binning used in Fig. 13 is $0.25 \AA$ per bin.)

The metallicity distribution of the galactic GCs is bimodal, with peaks at $[\mathrm{Fe} / \mathrm{H}]$ of -1.6 and -0.6 , using the data of Zinn (1985). The overall mean $[\mathrm{Fe} / \mathrm{H}]$ is -1.3 , and the root mean square (rms) dispersion is $\sigma=0.6$ dex, which translates into $\approx 1.4 \AA$ in the $\mathrm{Mg} b$ index. The main metalpoor peak at $[\mathrm{Fe} / \mathrm{H}]=-1.6$ in the metallicity histogram of galactic GCs is approximately Gaussian with a dispersion of about 0.3 dex.

The Mg $b$ histogram for the M87 GC system has a FWHM comparable to that of the galactic GC system, but the mean $\mathrm{Mg} b$ index corresponds to a much higher metallicity. The mean value of $\mathrm{Mg} b$ in the M87 GCs is $2.28 \AA$ (with a formal value for $\sigma$ of $1.16 \AA$ ) and the median value is $2.18 \AA$. Based on Table 3, these correspond to $[\mathrm{Fe} / \mathrm{H}] \approx-0.85$ dex with $\sigma \approx 0.5 \mathrm{dex}$. A comparison with previous determinations of these parameters will be given in $\S 7$.

It is clear that a single Gaussian model cannot fit the form of the histogram in Figure 13. To quantify this observation, we have applied a "mixture-modeling" algorithm known as the KMM test. The KMM test is an implementation of the "Expectation Maximization" algorithm of Dempster, Laird, \& Rubin (1977) and is described in detail by McLachlan \& Basford (1988). Use of the KMM test for estimating the significance of multiple (Gaussian) populations in univariate astronomical data sets has been explored by Ashman, Bird, \& Zepf (1994). The test does not involve binning of the data. Because of the great interest in bimodal metallicity distributions in giant elliptical galaxies (e.g., Ashman \& Zepf 1992; Whitmore et al. 1995; Geisler et al. 1996) and in order to limit the number of free parameters, we confine the tests to double versus single Gaussian models.

One must take care in performing and interpreting such multiple component fits. For instance, blind application of the KMM test to the entire $\mathrm{Mg} b$ distribution in Figure 13 finds that the double Gaussian model is to be preferred with a very high significance, with the peaks of the Gaussians located at 2.0 and $4.3 \AA$, i.e., it uses the second Gaussian to fit the high-Mg $b$ tail, containing only $14 \%$ of the GCs.

We wish to see whether the double Gaussian model is preferred for the main body of the histogram, however. If the tail is removed by excluding GCs with $\mathrm{Mg} b>4.0 \AA$, then the KMM test prefers the double Gaussian with a marginally significant probability of $87 \%$. The Gaussians in this case are centered at 1.46 and $2.73 \AA$, containing respectively $55 \%$ and $45 \%$ of the total number of GCs in this $\mathrm{Mg} b$ range. The common dispersion of the Gaussians is $\sigma=0.60$ $\AA$. If instead the clipping is done for $\mathrm{Mg} b>3.5 \AA$, the significance level shoots up to $97 \%$, and the peaks are at 1.29 and $2.50 \AA$, containing $48 \%$ and $52 \%$ of the GCs, respectively. The common dispersion here is $\sigma=0.50 \AA$. Thus, in neither of these cases would the two components be clearly distinguished in the histogram, as the separation between the Gaussians is just over $2 \sigma$. The high significance level returned by $\mathrm{KMM}$ in the latter case is likely due to the artificial boxiness caused by the overly zealous clipping (see the discussion by Ashman et al. 1994). We conclude that the actual significance level of the bimodality, with the tail excluded, is $85 \%-90 \%$. The following section revisits the bimodality issue in the context of the final $[\mathrm{Fe} / \mathrm{H}]$ distribution.

\section{SEPARATION OF AGE AND ABUNDANCE USING WORTHEY'S MODELS}

\subsection{Calibration of the Worthey Models}

We now proceed to a more quantitative treatment of the data. We use the models of Worthey (1994), assuming a single burst of star formation and a Salpeter initial mass function (IMF). The choice of IMF is not critical as long as the integrated light is dominated by the giants rather than by the main-sequence dwarfs. It is sufficiently difficult just to extract an abundance and an age for each M87 GC that we chose to avoid the complications of the additional parameters introduced by the models of Borges et al. (1995).

We will use the Worthey (1994) models to assign definite metallicities and ages to the M87 GCs. First we need to rescale the predictions of these models to reproduce the metallicities we have adopted for the galactic GCs, namely those of Zinn (1985). We define a canonical metallicity (henceforth $[\mathrm{Fe} / \mathrm{H}]_{\mathrm{W}}$ ) as the metallicity predicted by the Worthey (1994) model for a fixed age of 12 billion years using a $\chi^{2}$ minimization to the $\mathrm{Mg} b, \mathrm{NaD}, \mathrm{Fe} \lambda 5270$, and Fe $\lambda 5335$ indices together with their appropriate errors. The $\mathrm{H} \beta$ index is ignored here. Tests using simultaneous fitting for both age and metallicity with the Worthey (1994) model grid show that $[\mathrm{Fe} / \mathrm{H}]_{\mathrm{W}}$ differs little from that of a twoparameter fit.

Next we rescale the $[\mathrm{Fe} / \mathrm{H}]_{\mathrm{W}}$ values inferred for the galactic GCs using the data in Table 3 with the Worthey (1994) models to produce agreement with the Zinn (1985) metallicities for the galactic GCs. This rescaled value is denoted as $[\mathrm{Fe} / \mathrm{H}]_{Z}$. Figure 14 shows $[\mathrm{Fe} / \mathrm{H}]_{Z}$ values from Zinn (1985) versus $[\mathrm{Fe} / \mathrm{H}]_{\mathrm{w}}$ from the Worthey models for the galactic globular clusters and indicates the scaling required. A linear transformation, $[\mathrm{Fe} / \mathrm{H}]_{Z}=0.760$ $\times[\mathrm{Fe} / \mathrm{H}]_{\mathrm{w}}-0.265$, is satisfactory. The new data for the galactic GCs that were actually used to derive the fit are shown as filled circles, and open circles denote the data of Burstein et al. (1984). The symbols representing clusters in common between the two samples are connected by lines.

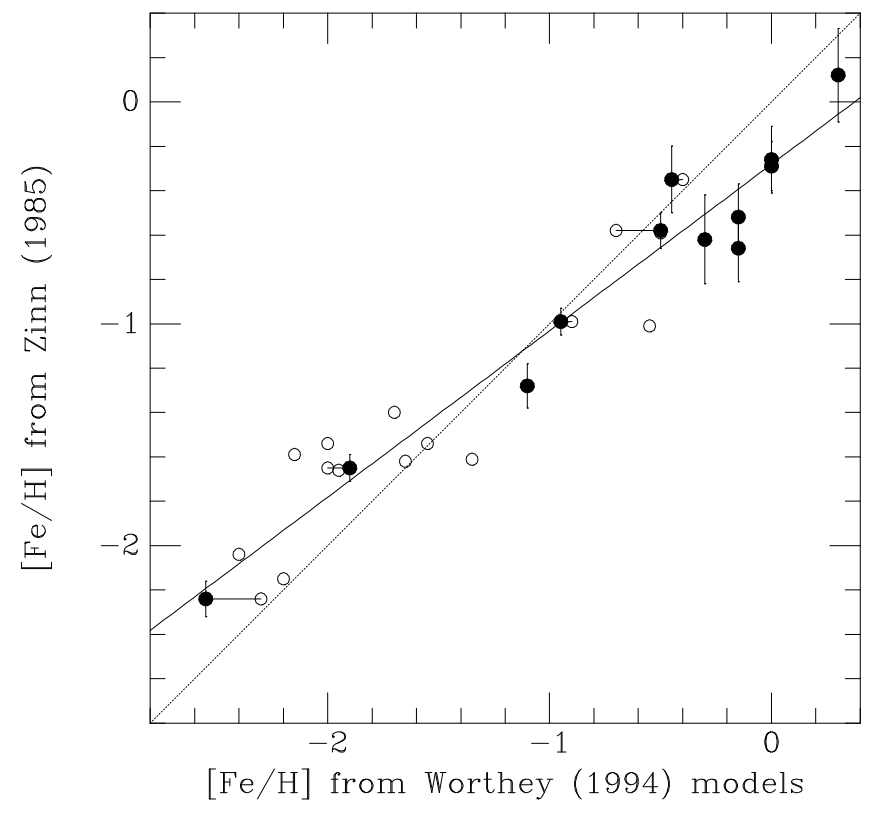

FIG. 14.-Metallicity from Zinn (1985) of the galactic globular clusters in our calibration sample is shown as a function of that derived for them using the Worthey (1994) models and their observed indices. 
The agreement between the predictions of the Worthey models and the data for the M87 and galactic GCs is shown in Figures 15-19. These figures display only the behavior of the M87 GCs, but we have already demonstrated in Figures $2-11$ that the behavior of the galactic globular clusters follows the median line of the M87 GCs. Figures 15, 16, and 17 are index-index plots ( $\mathrm{NaD}$ vs. $\mathrm{Mg} b, \mathrm{Fe} \lambda 5270$ vs. $\mathrm{Mg} b$, and $\mathrm{H} \beta$ vs. $\mathrm{Mg} b$ ) onto which the predictions of the Worthey (1994) models have been overlaid. Figure 18 dis-

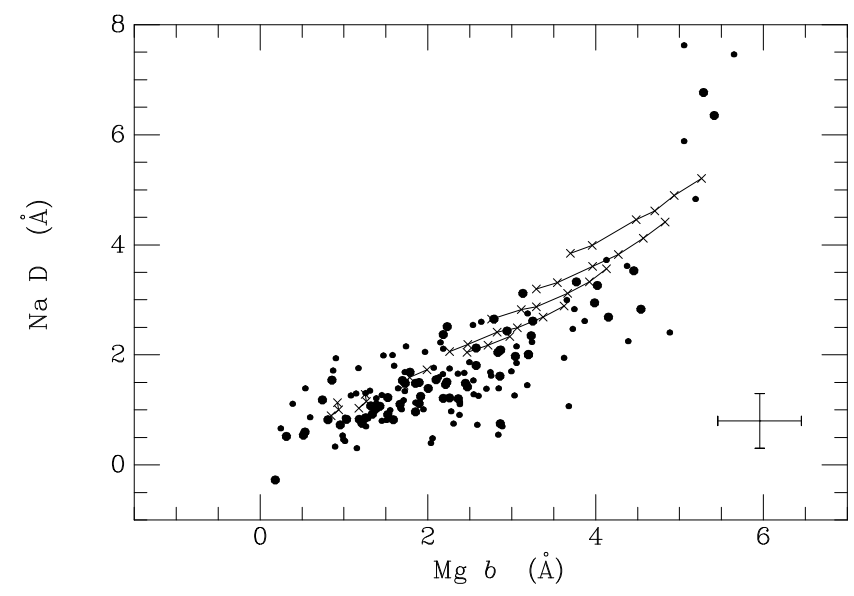

FIG. 15.- NaD indices for $150 \mathrm{M} 87 \mathrm{GCs}$ are shown as a function of their $\mathrm{Mg} b$ indices with the predictions of the Worthey (1994) models overlaid. Each line connects models of fixed metallicity with varying ages.

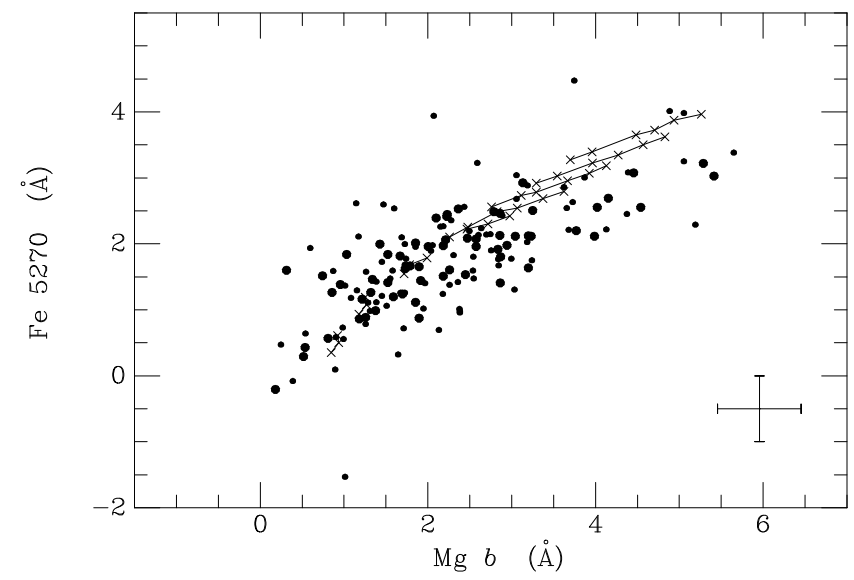

FIG. 16.-Same as Fig. 15 but for the Fe $\lambda 5270$ indices

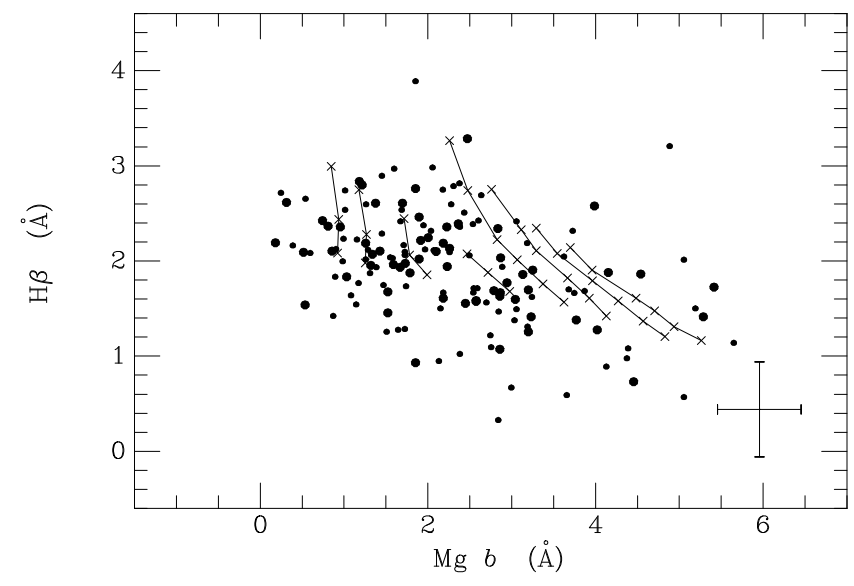

Fig. 17.-Same as Fig. 15 but for the $\mathrm{H} \beta$ indices

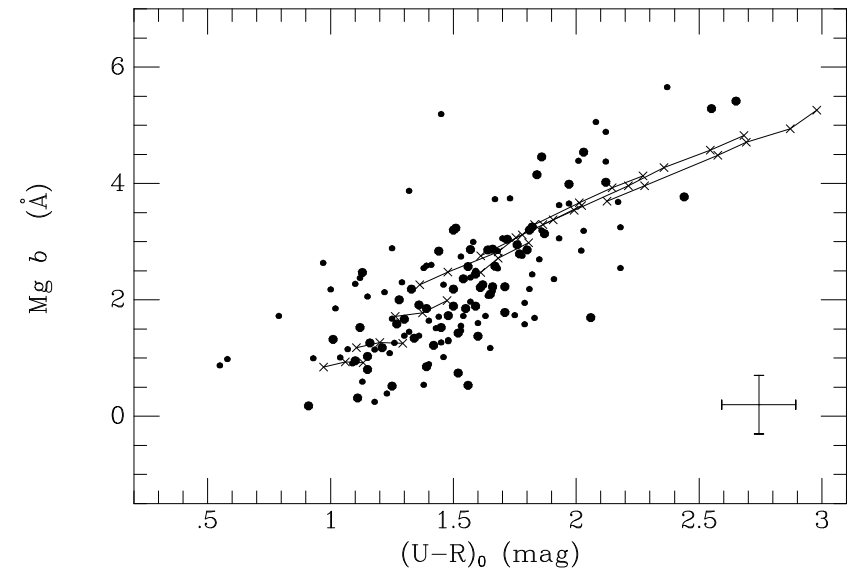

FIG. 18.- $\mathrm{Mg} b$ indices for the M87 GCs are shown as a function of $(U-R)$ color. The Worthey (1994) models are overlaid as in Fig. 15.

plays $\mathrm{Mg} b$ versus the $(U-R)$ color with the predictions of the Worthey models, while Figure 19 does the same for the $\mathrm{H} \beta$ index. The Worthey (1994) models range from 3 times solar to $1 / 100$ solar metallicity (which values have to be rescaled to our adopted Zinn scale) with ages of 2, 3, 5, 8, 12, and $17 \mathrm{Gyr}$. The metal-poor models were only computed for ages of 8,12, and $17 \mathrm{Gyr}$, but that does not matter, as no M87 GC turns out to be younger than $6 \mathrm{Gyr}$. The sequence of models of a fixed metallicity are connected by a line, and the prediction for each age is given by an " $\mathrm{x}$ " along the line.

One has the overall impression from looking at these figures that the Worthey (1994) models are a reasonable representation of the data. Note the very different behavior predicted for $\mathrm{H} \beta$ as a function of age (and metallicity) than for the $\mathrm{NaD}$ and $\mathrm{Mg} b$ indices, as supported by the data for both the M87 and the galactic GCs. The ability of the Worthey models to reproduce the mean behavior of the $\mathrm{H} \beta$ index at all values of the $\mathrm{Mg} b$ index implies that the assumptions made regarding the contribution of the horizontal branch (HB) and of more advanced stages of stellar evolution to the total integrated light in these models are basically correct. These assumptions include that the standard HB for metal-rich clusters is a red clump, and there is no extended blue HB such as was recently found in several metal-rich galactic GCs by Rich et al. (1997). Over the wavelength range considered here, if such an extended blue HB is present in metal-rich GCs, its contribution to the integrated light must be small.

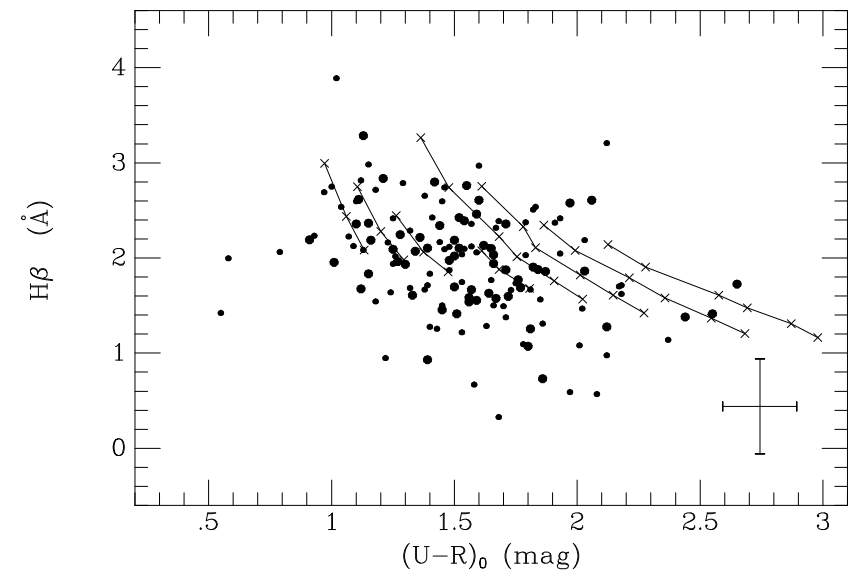

FIG. 19.- Same as Fig. 18 but for the $\mathrm{H} \beta$ indices 
The very strong NaD lines seen in six of the M87 GCs are partially reproduced by the most metal-rich of the Worthey models, but not completely.

Figure 16 shows the most glaring failure of the Worthey (1994) models, in that the Fe 25270 indices of the M87 GCs are always smaller than predicted by the models at the metal-rich (high- $\mathrm{Mg} b$ ) end. This is reminiscent of the problems encountered by Worthey, Faber, \& Gonzalez (1992) in trying to match the indices of elliptical galaxies.

We thus have some, although not perfect, confidence in the validity of the Worthey (1994) models for the integrated light of stellar systems, and hence in the ages and metallicities derived by applying them to our data on the M87 GCs.

The Worthey (1994) models were extrapolated to $1 / 200$ solar metallicity. Taking into account the rescaling of the Worthey abundance scale, the minimum possible metallicity returned by the fit is $[\mathrm{Fe} / \mathrm{H}]_{Z}=-2.2$ dex, while 1.3 times solar is the maximum. Also the metal-poor models were extrapolated to an age of $5 \mathrm{Gyr}$, which is the minimum possible age for a metal-poor model. The age range returned by the fit is $5-17$ Gyr.

Figure 20 shows the metallicity distribution $\left([\mathrm{Fe} / \mathrm{H}]_{Z}\right)$ for the sample of $150 \mathrm{M} 87 \mathrm{GCs}$. The mean $[\mathrm{Fe} / \mathrm{H}]_{Z}$ is -0.98 dex, with $\sigma=0.5$ dex, while the median $[\mathrm{Fe} / \mathrm{H}]_{Z}$ is -0.95 dex. The rms error in $[\mathrm{Fe} / \mathrm{H}]$ due only to measurement errors for the $\mathrm{Mg} b$ index is 0.22 dex for an object with QSNR such that it just gets into the abundance sample. This abundance error is actually slightly degraded by adding in the $\mathrm{Na}$ and $\mathrm{Fe}$ indices because of their smaller range (with the same measurement error). Overlaid on this metallicity distribution is the histogram for the galactic GCs represented by a solid line with data from the on-line compilation of Harris (1996), which is on the same metallicity scale (that of Zinn 1985) that we have adopted here. Just as in Figure 13, the M87 GC system shows a broad peak with a possible hint of multiple components. An artificial pileup of five objects at each of the extremes of the allowed range for metallicity, -2.2 and $+0.1 \mathrm{dex}$, is seen in Figure 20, while in Figure 13 these objects are shown more correctly as tails in the distribution of the $\mathrm{Mg} b$ indices. It is clear from Figures 3, 9, and 13 that the objects at the upper limit of $[\mathrm{Fe} / \mathrm{H}]$ returned by our fit are in fact more metalrich than any of the galactic GCs.

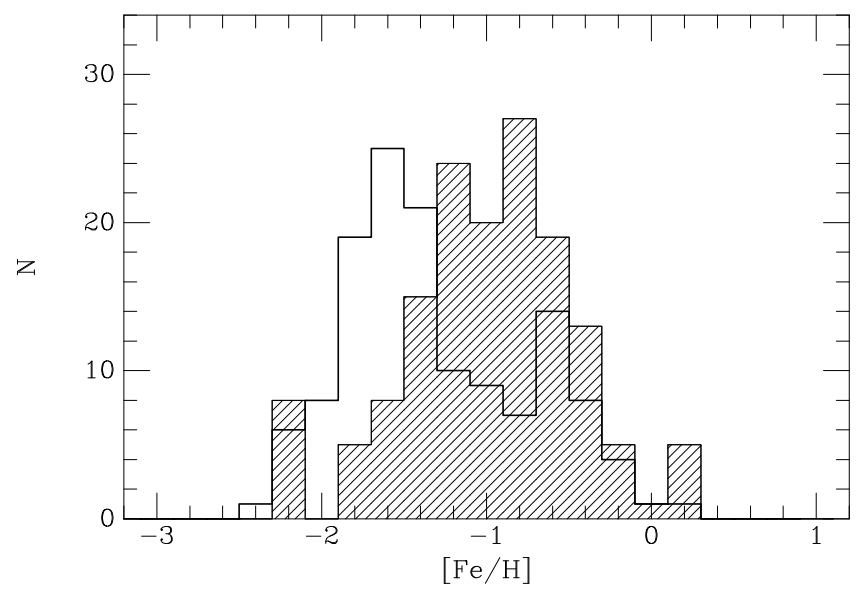

Fig. 20.-Histogram of metallicity for 150 M87 GCs is shown (hatched). The solid line represents that of the galactic GC system from Harris (1996).
We performed the KMM test on the final list of $[\mathrm{Fe} / \mathrm{H}]_{Z}$ values, excluding those artificially piled up at the extremes. The test results indicate that the double Gaussian model is preferred over the single one at the $89 \%$ significance level. The two "subpopulations" in this model are located at -1.3 and -0.7 dex and contain $40 \%$ and $60 \%$ of the total, respectively. The common dispersion is $\sigma=0.3$ dex. These results are similar to those found for the $\mathrm{Mg} b$ distribution, namely that there is marginal evidence, at the $\$ 90 \%$ level, for bimodality in the metallicity distribution. The locations of the metallicity "peaks" found here correspond well with those inferred from the $\mathrm{Mg} b \mathrm{KMM}$ test results.

For the halo of M87, the Dressler et al. (1987) measurement of the Lick indices can be transformed into $[\mathrm{Fe} / \mathrm{H}]$ using the Worthey models. Angeletti \& Giannone (1997) do this, extrapolating the measurements made on the nucleus to a projected radius of $200^{\prime \prime}$, and find $[\mathrm{Fe} / \mathrm{H}]_{Z}=-0.3 \mathrm{dex}$, where we have transformed their value to our adopted metallicity scale. This is about a factor of 4 higher than the metallicity of the M87 GCs at a similar projected radius. All photometric studies of the M87 GC system, beginning with the work of Strom et al. (1981), have found that the mean color of the GCs at a particular $R$ is significantly bluer than the light of the halo of M87 itself at that projected radius. This color difference is produced by the difference in mean metallicity discussed above.

\subsection{Determination of the Age Distribution of the M87 Galactic Clusters}

We intend to apply the Worthey (1994) models as described above to predict both the age and metallicity of a M87 GC from the full set of indices, $\mathrm{Mg} b, \mathrm{NaD}, \mathrm{Fe} \lambda 5270$, $\mathrm{Fe} \lambda 5335$, and $\mathrm{H} \beta$. Since the age discrimination is coming primarily from the $\mathrm{H} \beta$ index, the age is extremely sensitive to errors in that index. We therefore calculate the ages using the median lines for $\mathrm{H} \beta$ rather than the values of the $\mathrm{H} \beta$ index for each individual object. In effect, we are calculating the median age as a function of metallicity, since the $\mathrm{H} \beta$ lines are determined with respect to the metal indices.

First we test the scheme using the combined sample of galactic globular clusters, our data plus that of Burstein et al. (1984). The histogram of ages for this sample of 23 galactic GCs is given in Figure 21. The hatched area denotes our sample of galactic GCs, while the open area plus the

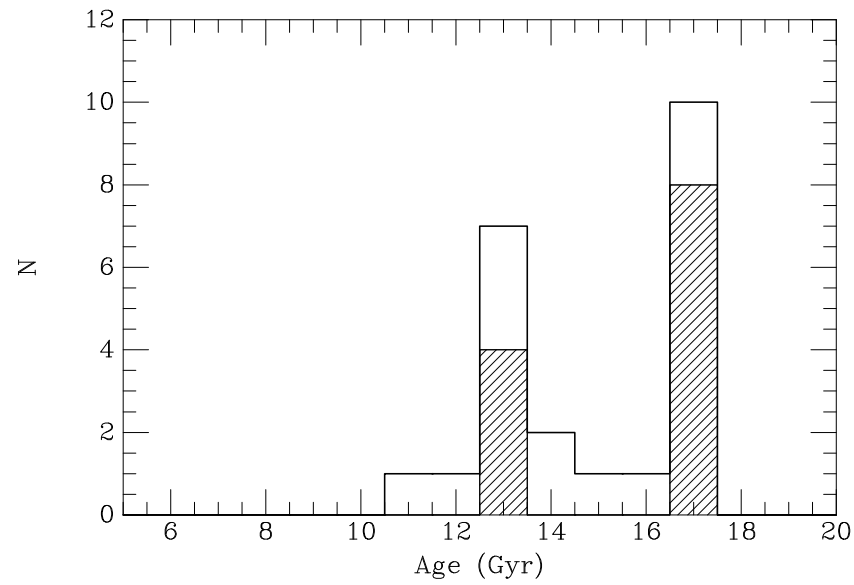

FIG. 21.-Histogram of ages derived for a sample of galactic globular clusters using the Worthey (1994) models applied just to the data presented here (hatched) and to our data combined with the sample of Burstein et al. (open). 


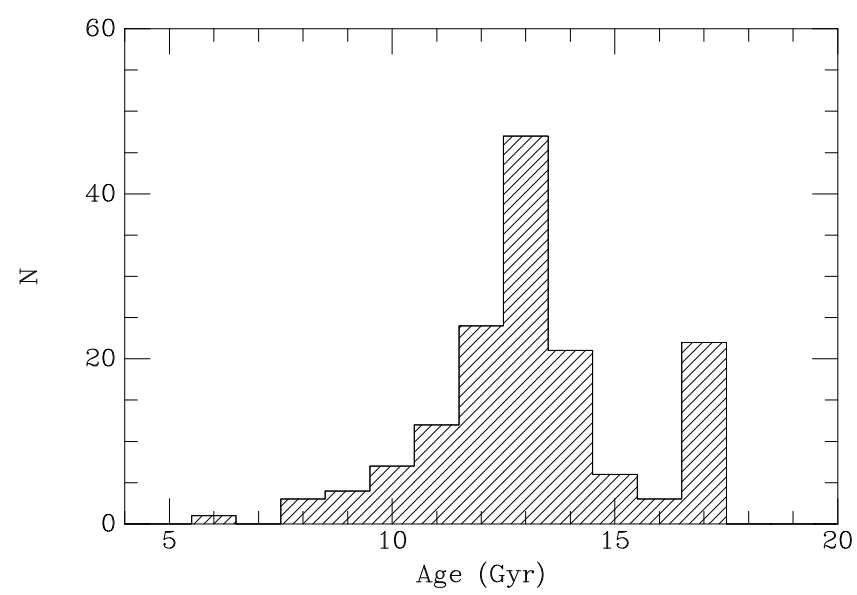

Fig. 22.- - Histogram of ages derived for the M87 GC system using the Worthey (1994) models applied to the data presented here.

hatched area denotes the merged sample. (For all the galactic GCs with $E[B-V]>0.4 \mathrm{mag}$, the $\mathrm{NaD}$ index was not used, as it is heavily contaminated by interstellar absorption.) A number of galactic GCs piled up at the upper limit of the fit, 17 Gyr. Figures 10, 17, and 19 demonstrate the problem, namely the metal-rich galactic GCs tend to lie below the median line determined by the much larger M87 sample, i.e., their $\mathrm{H} \beta$ indices appear weaker than those predicted even by Worthey's (1994) oldest models, while the metal-poor ones tend to lie above it, producing the two peaks in the histogram of age shown in Figure 21. Thus, at least formally, we find the metal-rich galactic GCs to be older than the metal-poor ones.

The median age of the galactic GCs is 15 Gyr. While no special credence should be placed in this value, it is well within the range of ages normally ascribed to the galactic GCs, and hence we believe that this procedure will give an accurate determination of the relative age of the M87 and galactic GC systems.

We now apply the Worthey (1994) models to our M87 data. We averaged the indices from the two weaker $\mathrm{Fe}$ blends $\lambda 5270$ and $\lambda 5335$, using the average $\mathrm{Fe}$ plus $\mathrm{NaD}$ and $\mathrm{Mg} b$ indices (with their appropriate errors) and the $\mathrm{H} \beta$ median lines. The result of this process is the age distribution for the M87 GC system shown in Figure 22. The pileup at $17 \mathrm{Gyr}$ is artificial and is due to the maximum age returned by the fitting process. The mean age is $13.2 \mathrm{Gyr}$ with a $1 \sigma$ deviation of $2.2 \mathrm{Gyr}$, and the median age is 13 Gyr. This is identical to within the errors to the age of the galactic GC system obtained above.

We next assess the impact of observational errors in producing the spread seen in Figure 22. The $1 \sigma$ error in the $\mathrm{H} \beta$ index for a M87 GC with the minimum QSNR to get into our abundance sample was given in $\S 3.3$ as $0.5 \AA$, while the rms deviation of the 148 points around the median line for the $\mathrm{H} \beta$ index is $0.55 \AA$. (The spectra of two of the M87 GCs do not reach blue enough to allow measurement of the $\mathrm{H} \beta$ index.) Increasing the $\mathrm{H} \beta$ index by $0.5 \AA$ reduces the derived age from 13 to $6 \mathrm{Gyr}$, while decreasing that index by the same amount increases the age from $13 \mathrm{Gyr}$ to the upper limit of the fits at $17 \mathrm{Gyr}$. Given our sample size, the uncertainty in the median line at any point is $\approx 0.045 \AA$. A change of this size in the $\mathrm{H} \beta$ index changes the deduced age by $\approx 0.9 \mathrm{Gyr}$. Thus while observational errors seriously impact the age of an individual GC inferred from its measured $\mathrm{H} \beta$ index, use of the median line for the $\mathrm{H} \beta$ index should produce a good estimate of the age.

There is no evidence for a substantial population of M87 GCs younger than 12 Gyr. The median age and the age distribution of the M87 GCs and of the galactic GCs are identical.

Table 4 gives the age estimates for the M87 GCs divided into six metallicity bins, each containing 25 objects. There is no sign of a variation in age with metallicity. One should note, however, that the galactic globular clusters emerge from the above analysis with an age distribution such that formally the metal-rich galactic GCs are older than the metal-poor ones. We do not understand the cause of this. If it arises from an error in Worthey's (1994) models, then correcting this error might make the metal-rich M87 GCs somewhat younger than the metal-poor ones.

\subsection{Correlations of Velocity Dispersion with Metallicity}

We have looked for a correlation between velocity dispersion and metallicity among the M87 GCs in our sample, combining the abundances given here with the $v_{r}$ of Paper I. Such a correlation is predicted by various theories of globular cluster formation, particularly if one chooses to believe that the metal-rich population of GCs is a "disk population," while the metal-poor GCs represent the halo. In this case one would expect the metal-poor GCs to show a larger velocity dispersion, while the metal-rich ones, with their smaller velocity dispersion, might show rotation, as is seen in the Galaxy (Zinn 1985).

Subdividing the sample of 150 M87 GCs into halves, and then into thirds, does not show the expected effect. If anything, the velocity dispersion of the metal-poor GCs is smaller than that of the metal-rich ones.

This issue is complicated by exactly what one adopts for the rotation curve for the stellar component of M87 and for the M87 GCs (see Paper I).

\section{COMPARISON WITH PREVIOUS STUDIES AND WITH STUDIES OF NGC 1399}

\subsection{Comparison with Previous Studies of M87}

As discussed earlier, there have been many photometric studies of the M87 GC system, both from the ground and

TABLE 4

Age Estimates by Metallicity for the M87 Globular Clusters

\begin{tabular}{ccccc}
\hline \hline Bin & Median $[\mathrm{Fe} / \mathrm{H}]_{Z}(\mathrm{dex})$ & Mean Age $(\mathrm{Gyr})$ & Median Age $(\mathrm{Gyr})$ & $\sigma_{\text {Age }}(\mathrm{Gyr})$ \\
\hline $1 \ldots \ldots$ & -1.75 & 14.6 & 14.0 & 1.7 \\
$2 \ldots \ldots$. & -1.29 & 13.8 & 13.0 & 1.7 \\
$3 \ldots \ldots$ & -1.02 & 12.6 & 13.0 & 2.0 \\
$4 \ldots \ldots$ & -0.84 & 11.8 & 12.0 & 1.9 \\
$5 \ldots \ldots$ & -0.68 & 12.6 & 13.0 & 1.5 \\
$6 \ldots \ldots$ & -0.30 & 13.6 & 13.0 & 3.1 \\
\hline
\end{tabular}


from $H S T$, as well as initial spectroscopic studies of small samples. The mean metallicity we have derived for the M87 GC system, $-0.98 \mathrm{dex}$, with $\sigma=0.5 \mathrm{dex}$ on the scale of Zinn (1985) is in good agreement with the earlier estimates summarized in Table 5.

The beautiful X-ray data from Ginga (Koyama, Takano, \& Tawara 1991) and from ASCA (Matsumoto et al. 1996) extend from the nucleus to $R=40^{\prime}$ and show definite small radial gradients in $\mathrm{O}, \mathrm{Si}, \mathrm{S}$, and $\mathrm{Fe}$, with the gradient in $\mathrm{O}$ being the weakest. The abundances they derive for the hot X-ray-emitting gas are significantly higher than those inferred from the M87 GC system and are comparable to those of the mean of the M87 stellar halo. This might be due to the buildup of heavy elements in the gas since the initial formation of the M87 GCs through mass loss from the M87 halo stars.

The subject of radial gradients in the M87 GC system (see the review by Harris 1991) has been quite controversial. Although Strom et al. (1981) claimed to detect a radial gradient in the mean broadband colors of the M87 GCs, this was not reproduced by subsequent, presumably more accurate, CCD-based studies by Cohen (1988) and by Couture et al. (1990). However, Lee \& Geisler (1993) using Washington photometry claimed to detect a radial gradient of a factor of 4 in mean abundance over the range in projected radius of their observations, $60^{\prime \prime}-500^{\prime \prime}$.

It is clear from our work that there is a radial gradient in mean abundance in the M87 GC system, but that the dispersion in metallicity at all radii is so large that its easy to lose in the observational errors. It is a tribute to the metallicity sensitivity of the ultraviolet colors used in the original photographic survey of Strom et al. (1981) and of the Washington photometric system used by Lee \& Geisler (1993) that they detected this gradient. The large size of these two samples and their large range in radial coverage also contributed to detection of these gradients. The calibration of photometric indices versus metallicity for these two photometric systems is derived using our $[\mathrm{Fe} / \mathrm{H}]_{Z}$ values for the M87 GCs together with published photometry for these objects in Appendix C.

The exact size of the radial gradient of abundance within the M87 GC system is still not well determined, but the existence of a gradient is now beyond doubt.

Meanwhile the high-precision HST photometric surveys of the M87 GC system by Whitmore et al. (1995) and by Elson \& Santiago (1996a, 1996b) have as their major new result the claim that the abundance distribution of the M87 GCs is bimodal, with peaks that agree between these two studies at $(V-I) \approx 0.95$ and $\approx 1.20 \mathrm{mag}$. Using the transformation of Couture et al. (1990), which is on the Zinn (1985) abundance scale, these colors correspond to [Fe/ $\mathrm{H}]=-1.3$ and 0.0 dex. They do not agree well with the histogram of abundances for the M87 GC system we obtained shown in Figure 20. Use of the transformation of
Kissler-Patig et al. (1998), which was developed for very red objects, suggests the upper peak has $[\mathrm{Fe} / \mathrm{H}]-0.5 \mathrm{dex}$, much closer to what we see in our M87 GC metallicity distribution. The radial gradients in the M87 GC system also need to be taken into account here, as the HST studies are heavily biased toward the center of M87 due to the small spatial field of the wide-field and planetary camera (WFPC), while our analysis covers a much larger range in projected radius.

\subsection{Comparison with Results for NGC 1399}

The only other giant elliptical galaxy whose GC system has been studied in some detail is NGC 1399, the cD galaxy in the Fornax cluster. There have been many broadband photometric studies (see, for example, Bridges, Hanes, \& Harris 1991 and a study using the Washington system by Ostrov, Geisler, \& Forte 1993 that augments an earlier effort by Geisler \& Forte 1990). Very recently Kissler-Patig et al. (1998) presented an initial spectroscopic survey of a small sample of GCs in NGC 1399 using LRIS at Keck. The available results regarding the abundances in the NGC 1399 GC system are quite similar to what we find in the M87 GC system. The mean [Fe/H] for the NGC $1399 \mathrm{GC}$ system is $-0.90 \pm 0.2$ dex, with a spread ranging from solar to very metal-poor. Ostrov et al. (1993) report a small radial gradient.

\section{IMPLICATIONS FOR GALAXY AND GLOBULAR CLUSTER FORMATION}

Harris (1991) reviewed the many theories that have been advanced to explain the formation of globular clusters and their confrontation with the mounting body of known facts about these systems. In recent years, more advanced numerical simulations of galaxy mergers (e.g., Barnes \& Hernquist 1992) and isophotal evidence such as shell structures (e.g., Quinn 1984) have convinced many people that mergers of spiral galaxies may play an important role in the formation and evolution of elliptical galaxies. Ashman \& Zepf (1992, 1997) have emphasized the possible role of mergers on the formation of GC systems and have claimed that they can reproduce the high $S_{N}$ values (a parameter that characterizes the number of globular clusters per unit halo luminosity) seen in systems such as M87 under such circumstances.

Our work presents another set of constraints for these models. The constraints we have placed on the radial velocity distribution (see Paper I), the metallicity distribution, the radial abundance gradients, and the age distribution within the M87 GC system suggest that mergers, if important, all happened near the beginning of the collapse of the Virgo galaxy cluster. Everything is so well organized, and so continuous with the properties of less luminous elliptical galaxies, that mergers of fully developed galaxies to form elliptical galaxies seem quite unlikely. Global correlations

TABLE 5

Comparison of Mean [Fe/H] For M87 Globular Clusters

\begin{tabular}{|c|c|c|c|c|c|}
\hline Reference & $\begin{array}{c}\langle[\mathrm{Fe} / \mathrm{H}]\rangle \\
(\mathrm{dex})\end{array}$ & $\begin{array}{c}\sigma \\
(\mathrm{dex})\end{array}$ & Reference & $\begin{array}{c}\langle[\mathrm{Fe} / \mathrm{H}]\rangle \\
(\mathrm{dex})\end{array}$ & $\begin{array}{c}\sigma \\
(\operatorname{dex})\end{array}$ \\
\hline Photometry & (ground based) & & Spectroscopy & & \\
\hline Cohen 1988 & -1.0 & & Mould et al. $1990 .$. & -1.0 & $\ldots$ \\
\hline Couture et al. 1990 ...... & -1.1 & 0.6 & Brodie \& Huchra $1991 \ldots \ldots$. & -0.9 & $\ldots$ \\
\hline Lee \& Geisler $1993 . . . .$. & -0.85 & 0.65 & This Paper................... & -0.95 & 0.5 \\
\hline
\end{tabular}


such as that between the metallicity of the GC system of an elliptical galaxy and its luminosity, first pointed out by van den Bergh (1975), are now even stronger.

Our major new contribution to this process is that we have established the timescale for the process of the assembling of the GC system of a galaxy like M87; it seems to have been less than 2 Gyr. This is a time span comparable to the age range believed to exist among the galactic GCs (see VandenBerg, Bolte, \& Stetson 1996).

Theories where the GC system of the central cD galaxy in a cluster of galaxies is formed by accreting GCs from neighboring galaxies through orbital effects, such as that advocated by Muzzio (1987), can be ruled out on the basis of the extended timescale for GC formation. Formation of GCs in the cooling flow around the central cD in clusters of galaxies advanced by Fabian, Nulsen, \& Canizares (1984) can also be ruled out for similar reasons.

Another important point to note where our work establishes a new constraint is that the relative abundances of $\mathrm{Fe}$, $\mathrm{Na}$, and $\mathrm{Mg}$ appear to be changing together in the M87 GC system in the same way as in the galactic globulars. In other words, to first order the nucleosynthesis history appears to have been the same in these two GC systems. The same is true for the M31 GC system (Cohen, Djorgovski, \& McCarthy 1997). The details of chemical evolution of GCs depend on many complex and poorly understood phenomena. These include the process of galaxy formation, the halo formation process, star formation within the protoglobular cluster, the formation of supernovae, the nuclear burning within supernovae and the dispersal of their ejecta, the processes of stellar evolution, etc. Somehow all of this happened so as to produce very similar and very reproducible trends in the oldest objects we can study. These include very distant objects at high redshift in the young universe that we perceive through the QSO absorption lines that they produce ( $\mathrm{Lu}$ et al. 1996) as well as old objects in the local universe, such as the M87 GC system, the galactic GC system, or the galactic halo.

It is very reassuring that in some sense the laws of physics work over such enormous ranges in distance and time. Obviously as one looks in more detail, as is possible within our own Galaxy, one begins to see finer and finer effects. High-precision studies of field halo stars, as well as of stars in galactic globular clusters, have established that in our Galaxy there are a number of prominent changes in abundance ratios, such as the increase of $\alpha$-element abundances relative to $\mathrm{Fe}$ in stars with $[\mathrm{Fe} / \mathrm{H}]$ between -2 dex and solar abundance, as reviewed by Wheeler, Sneden, \& Truran (1989). This particular trend is believed to be due to the time delay involved for Type Ia supernovae to become effective sources of heavy elements. These trends have been further extended to even lower metallicity by several groups, most recently McWilliam et al. (1995). But the gross trends appear to be universal. We can now examine in considerable detail the brightest early-type supergiants in the nearest Local Group galaxies (McCarthy et al. 1995; Monteverde et al. 1997) to find the same gross trends repeated.

\section{SUMMARY}

In Paper I we used radial velocity data to isolate a sample of 205 bona fide members of the M87 globular cluster system. Here we have selected a subset of this sample for more detailed analysis on the basis of the signal-to-noise ratio of their spectra. This sample includes 162 spectra of 150 M87 GCs.

We used the Lick indices to measure the strength of the stronger absorption line features because of the extensive database available for stars and the integrated light of stellar systems. We included $\mathrm{Mg} b, \mathrm{Fe} \lambda 5270, \mathrm{Fe} \lambda 5335$, $\mathrm{NaD}$, and $\mathrm{TiO}_{1}$, as well as the $\mathrm{H} \beta$ index, and added an index for $\mathrm{H} \alpha$.

First, we qualitatively compared the behavior of the M87 GCs and the galactic GCs. We combined new scans of 12 galactic GCs with the data of Burstein et al. (1984) for a total sample of 23 galactic GCs. A study of the index-index plots and of the index-broadband $(U-R)$ color plots shows that the metallicity range spanned by the M87 GC system is similar to that of the galactic GCs, but the former has a metal-rich tail extending to significantly higher metallicity than one finds among the galactic GCs.

There are six M87 GCs with extremely strong NaD indices. The NaD indices for the galactic GC system show definite evidence for the influence of interstellar absorption.

The relative abundances of $\mathrm{Fe}, \mathrm{Mg}$, and $\mathrm{Na}$ in the M87 GCs follow similar trends to those of the galactic GCs. To first order, the nucleosynthesis history in the M87 GC system appears to have been the same as in the Milky Way GC system. The behavior of the Balmer lines is also quite similar, implying a similar mean age and age distribution in the two systems.

There is a small radial gradient in the metal line indices in the sense of a decline in metallicity as $R$ (projected radius) increases, but there is no detectable radial change in the $\mathrm{H} \beta$ index. No dependence of dispersion of radial velocity with metallicity was detected.

To make the results more quantitative, we used the Worthey (1994) models for the integrated light of stellar systems assuming a single burst of star formation and a Salpeter initial mass function. We adopted the metallicity scale of Zinn (1985) for the galactic GCs. We checked the validity of the Worthey (1994) models and normalized their metallicity scale to the Zinn scale by analyzing the indices of the galactic GCs.

The age dependence comes almost entirely from the Balmer lines. In consideration of the observational errors, we combined the measured metal line indices for each object with the median lines for the $\mathrm{H} \beta$ index, rather than with the individually measured values for each GC, to derive the age of each galactic GC. The result is a median age of $15 \mathrm{Gyr}$ for the sample of 23 galactic GCs.

We then applied this formalism to the M87 GC system. The median age for the $150 \mathrm{M} 87 \mathrm{GCs}$ is $13 \mathrm{Gyr}$, with $\sigma=2$ Gyr. The median metallicity is $[\mathrm{Fe} / \mathrm{H}]_{Z}=-0.95$ dex with $\sigma=0.5 \mathrm{dex}$, in good agreement with previous work. There is no obvious bimodality in the abundance distribution, but the peak of the distribution is very broad. Moreover, application of the KMM test to the abundance distribution finds marginal evidence for bimodality, with peaks near $[\mathrm{Fe} / \mathrm{H}]_{Z} \sim-1.3$ and -0.7 . We compared these results to analyses of the GC system of NGC 1399, the cD giant elliptical galaxy at the center of the Fornax cluster, to find very similar properties for the two systems.

The major new contributions we have made to this field are twofold. The first is the establishment of the age distribution for the M87 GCs. The mean age for the M87 GCs is comparable to that of the galactic globular clusters, with a small dispersion $(\sigma=2 \mathrm{Gyr})$ about that value. The second 
concerns the details of chemical evolution and enrichment in GC systems. We have found that the same trends prevail among the abundances of $\mathrm{Fe}, \mathrm{Mg}$, and $\mathrm{Na}$ as occur in the galactic GC system. The former is a very powerful constraint, and we have briefly discussed the implications for theories of globular cluster formation.

The entire Keck/LRIS user community owes a huge debt to Jerry Nelson, Gerry Smith, Bev Oke, and many other people who have worked to make the Keck Telescope and LRIS a reality. We are grateful to the W. M. Keck Foundation, and particularly its late president, Howard Keck, for the vision to fund the construction of the W. M. Keck Observatory.

J. G. C. is grateful to NSF grant AST 96-16729 for support. J. P. B. is grateful to the Sherman Fairchild Foundation for support.

\section{APPENDIX A}

\section{NEW RADIAL VELOCITY MEASUREMENTS}

In the process of reanalysis of the spectra of the M87 GCs to prepare the final abundance sample, five M87 GCs were found for which the final spectra gave $v_{r}$ different from the values published in Paper I by more than $150 \mathrm{~km} \mathrm{~s}^{-1}$. In addition, Strom 682, which was not included in the $v_{r}$ sample of Paper I, now has a definite $v_{r}$. These values are listed in Table A1.

TABLE A1

New and Corrected Radial Velocities for M87 Globular Clusters

\begin{tabular}{|c|c|c|c|c|c|}
\hline Identification Number & $\begin{array}{c}v_{r}(\text { Paper I }) \\
\left(\mathrm{km} \mathrm{s}^{-1}\right)\end{array}$ & $\begin{array}{c}v_{r}(\text { new }) \\
\left(\mathrm{km} \mathrm{s}^{-1}\right)\end{array}$ & Identification Number & $\begin{array}{c}v_{r}(\text { Paper I }) \\
\left(\mathrm{km} \mathrm{s}^{-1}\right)\end{array}$ & $\begin{array}{c}v_{r} \text { (new) } \\
\left(\mathrm{km} \mathrm{s}^{-1}\right)\end{array}$ \\
\hline 5001 & 929 & 680 & 5020 & 1646 & 1450 \\
\hline $682 \ldots \ldots \ldots+\ldots, \ldots$ & & 1300 & $695 \ldots$ & 1869 & 1650 \\
\hline $1119 \ldots \ldots \ldots \ldots \ldots \ldots$ & 1997 & 1510 & $1353 \ldots \ldots$ & 2161 & 1980 \\
\hline
\end{tabular}

\section{APPENDIX B}

\section{NEW $U, R$ PHOTOMETRY}

A few of the M87 GCs in our sample do not have $U R$ photometry from Strom et al. (1981). Also the added sample of nuclear GCs in M87 has no published photometry. We therefore obtained a set of images in $U$ and in $R$ using COSMIC (Dressler 1993) at the prime focus of the $5 \mathrm{~m}$ Hale telescope at Palomar Observatory. Several COSMIC fields were required to cover the large angular field on the sky of the M87 GCs. Because of the overlap between fields, most of the M87 GCs for which colors are required appear on several of the fields and hence have multiple measurements. The total integration time/field is $100 \mathrm{~s}$ for $R$ and $1200 \mathrm{~s}$ for $U$. We used Strom et al.'s (1981) photographic photometry to define the zero points for each color. Table $\mathrm{B} 1$ lists our newly measured $U$ and $R$ magnitudes.

We thank C. Steidel for use of his $U$ filter.

TABLE B1

New Photometry for Globular Clusters in M87

\begin{tabular}{ccc|ccc|ccc}
\hline \hline Identity & $U$ & $R$ & Identity & $U$ & $R$ & Identity & $U$ & $R$ \\
\hline $5001 \ldots \ldots$ & $\ldots$ & 19.14 & $395 \ldots \ldots$ & $\ldots$ & 20.83 & $902 \ldots \ldots \ldots$ & 22.34 & 20.20 \\
$5002 \ldots \ldots$ & 21.29 & $\ldots$ & $423 \ldots \ldots$ & 22.84 & 20.81 & $917 \ldots \ldots$. & $\ldots$ & 20.73 \\
$5012 \ldots \ldots$ & $\ldots$ & 20.19 & $453 \ldots \ldots$ & $\ldots$ & 20.53 & $928 \ldots \ldots \ldots$ & 20.20 & 19.13 \\
$5015 \ldots \ldots$ & 20.83 & 18.71 & $519 \ldots \ldots$ & $\ldots$ & 20.79 & $968 \ldots \ldots \ldots$ & $\ldots$ & 20.87 \\
$5020 \ldots \ldots$ & 21.45 & 19.74 & $581 \ldots \ldots$ & $\ldots$ & 20.27 & $1032 \ldots \ldots$ & 21.40 & 20.21 \\
$5021 \ldots \ldots$ & $\ldots$ & 19.95 & $682 \ldots \ldots$ & $\ldots$ & 20.50 & $1067 \ldots \ldots$ & 22.06 & 19.90 \\
$5024 \ldots \ldots$ & 21.22 & 19.56 & $697 \ldots \ldots$ & 21.86 & 19.68 & $1091 \ldots \ldots$ & 22.07 & 20.26 \\
$5025 \ldots \ldots$ & 21.61 & 19.37 & $723 \ldots \ldots$ & $\ldots$ & 20.69 & $1101 \ldots \ldots$ & 22.16 & 20.57 \\
$5026 \ldots \ldots$ & 20.88 & 19.25 & $746 \ldots \ldots$ & 20.99 & 19.17 & $1110 \ldots \ldots$ & $\ldots$ & 20.73 \\
$5028 \ldots \ldots$ & 21.90 & 19.19 & $750 \ldots \ldots$ & 21.30 & 19.85 & $1113 \ldots \ldots$ & $\ldots$ & 20.43 \\
$248 \ldots \ldots$ & $\ldots$ & 19.77 & $770 \ldots \ldots$ & 21.87 & 20.31 & $1119 \ldots \ldots$ & $\ldots$ & 20.90 \\
$311 \ldots \ldots$. & 22.37 & 20.60 & $798 \ldots \ldots$ & $\ldots$ & 20.17 & $1180 \ldots \ldots$ & 20.93 & 19.39 \\
$324 \ldots \ldots$ & $\ldots$ & 20.34 & $871 \ldots \ldots$ & 22.56 & 20.64 & $1483 \ldots \ldots$ & $\ldots$ & 20.87 \\
$330 \ldots \ldots$ & $\ldots$ & 20.66 & $892 \ldots \ldots$ & 22.49 & 20.25 & $651 \ldots \ldots \ldots$ & $\ldots$ & 20.60 \\
\hline
\end{tabular}

\section{APPENDIX C}

\section{CALIBRATION OF $(U-R)$ AND WASHINGTON PHOTOMETRY AS A FUNCTION OF METALLICITY}

At the request of the referee, as an aid to future investigators we have derived a calibration of the $(U-R)$ photometry of Strom et al. (1981) in terms of our metallicities for the M87 GCs. A linear least-squares fit for the 133 M87 GCs in our sample 
with $(U, R)$ photometry from Strom et al. (1981) or from Table B1 and with $-2<[\mathrm{Fe} / \mathrm{H}]_{Z}<0$ dex gives $[\mathrm{Fe} / \mathrm{H}]_{Z}=0.81$ $\times(U-R)_{0}-2.25 \mathrm{dex}$, with a rms residual of 0.29 dex. Note that the dereddened color is used here; $E(U-R)=0.06 \mathrm{mag}$ for M87. A linear fit is adequate. This dispersion is consistent with the uncertainties of our abundances coupled with those of the photometry.

We have also done this for the Washington photometry of the M87 GCs by taking the $[\mathrm{Fe} / \mathrm{H}]$ values obtained by Lee \& Geisler (1993) for a sample of M87 GCs (Table 3 of their paper) and identifying these objects in the sample of Strom et al. (1981). Since there are several inconsistencies in Table 3 between the names of the fields in the table and the names of the fields on the charts of this paper, this was nontrivial. Because the area observed by Lee \& Geisler excludes most of the southern part of M87 and half of the East2 field (as identified on their charts) is beyond the area of the Strom et al. (1981) sample from which we picked our M87 GC candidates, there are only 38 objects in common. The resulting linear fit is $[\mathrm{Fe} / \mathrm{H}]_{Z}=1.01$ $\times[\mathrm{Fe} / \mathrm{H}]_{\mathrm{LG}}+0.16 \mathrm{dex}$ with a rms residual of 0.39 dex. This fit appears to fail at the highest metallicities. A quadratic fit gives only a slightly smaller dispersion.

Angeletti, L., \& Giannone, P. 1997, A\&A, 321, 343

Ashman, K. M., Bird, C. M., \& Zepf, S. E. 1994, AJ, 108, 2348

Ashman, K. M., \& Zepf, S. E. 1992, ApJ, 384, 50 Press)

Barnes, J. E., \& Hernquist, L. 1992, ARA\&A, 30, 705

Bridges, T. J., Hanes, D. A., \& Harris, W. E. 1991, AJ, 101, 469

Borges, A. C., Idiart, T. P., de Freitas Pacheco, J. A., \& Thevenin, F. 1995, AJ, 110, 2408

Brodie, J. P., \& Huchra, J. P. 1990, ApJ 362, 503

.1991, ApJ, 379, 157

Burstein, D., Faber, S. M., Gaskell, C. M., \& Krumm, N. 1984, ApJ 287, 586

Burstein, D., \& Heiles, C. 1984, ApJS, 54, 33

Chavez, M., Malgnini, M. L., \& Morossi, C. 1995, ApJ, 440, 210

Cohen, J. G. 1975, ApJ, 197, 117 1988, AJ, 95, 682

Cohen, J. G., Djorgovski, S. G., \& McCarthy, J. K. 1998, in preparation

Cohen, J. G., \& Ryzhov, A. 1997, ApJ, 486, 230 (Paper I)

Couture, J., Harris, W. E., \& Allbright, J. W. B. 1990, ApJS, 73, 671

Dempster, A. P., Laird, N. M., \& Rubin, D. B. 1977, J. R. Statist. Soc. B, 39, 1

Dressler, A. 1993, in Palomar Obs. Ann. Rep., 1993, 2

Dressler, A., et al. 1987, ApJ, 313, 42

Elson, R. A. W., \& Santiago, B. X. 1996a, MNRAS, 278, 617 1996b, MNRAS, 280, 971

Faber, S. M., Friel, E. D., Burstein, D., \& Gaskell, C. M. 1985, ApJS, 57, 711

Fabian, A. C., Nulsen, P. E. J., \& Canizares, C. R. 1984, Nature, 310, 733

Geisler, D., \& Forte, J. C. 1990, ApJ, 350, L5

Geisler, D., Lee, M. G., \& Kim, E. Y. 1996, AJ, 111, 1529

Gorgas, J., Faber, S. M., Burstein, D., Jesus Gonzalez, J., Courteau, S., \& Prosser, C. 1993, ApJS, 86, 153

Hanes, D. A., \& Brodie, J. P. 1986, ApJ, 300, 279

Harris, W. E. 1991, ARA\&A, 29, 543 . 1996, AJ, 112, 1487

Hobbs, L. M. 1974, ApJ, 191, 381

Kissler-Patig, M., Brodie, J. P., Schroder, L. L., Forbes, D. A., Grillmair, C. J., \& Huchra, J. P. 1998, AJ, in press

Koyama, K., Takano, S., \& Tawara, Y. 1991, Nature, 350, 135

\section{REFERENCES}

Kurucz, R. L. 1993, Kurucz CD-ROM 13, ATLAS9 Stellar Atmosphere Programs (Cambridge: Smithsonian Astrophysical Observatory)

Lee, M. G., \& Geisler, D. 1993, AJ, 106, 493

Lu, L. M., Sargent, W. L. W., Barlow, T. A., Churchill, C. W., \& Vogt, S. S. 1996, ApJS, 107, 475

Matsumoto, H., Koyama, K., Awaki, H., Tomida, H., Tsuru, T., Mushotzky, R., \& Hatsukade, I. 1996, PASJ, 48, 201

McCarthy, J. K., Lennon, D. J., Venn, K. A., Kudritzski, R. P., Puls, J., \& Navarro, F. 1995, ApJ, 455, L155

McLachlan, G. J., \& Basford, K. E. 1988, Mixture Models: Inference and Applications to Clustering (New York: Marcel Dekker)

McWilliam, A., Preston, G. W., Sneden, C. \& Searle, L. 1995, AJ, 109, 2757

Monteverde, M. I., Herrero, A., Lennon, D. J., \& Kudritzki, R. P. 1997, ApJ, 474, L107

Mould, J. R., Oke, J. B., de Zeeuw, P. T., \& Nemec, J. M. 1990, AJ, 99, 1823

Mould, J. R., Oke, J. B., \& Nemec, J. M. 1987, AJ, 93, 53

Muzzio, J. C. 1987, PASP, 99, 614

Oke, J. B., et al. 1995, PASP, 107, 307

Ostrov, P., Geisler, D., \& Forte, J. C. 1993, AJ, 105, 1762

Press, W. H., Flannery, B. P., Teukolsky, S. A., \& Vetterline, W. T. 1986, Numerical Recipes (Cambridge: Cambridge Univ. Press)

Quinn, P. J. 1984, ApJ, 279, 596

Rabin, D. 1982, ApJ, 261, 85

Racine, R., Oke, J. B., \& Searle, L. 1978, ApJ, 223, 82

Reed, B. C., Hesser, J. E., \& Shawl, S. J. 1988, PASP, 100, 545

Rich, R. M., et al. 1997, ApJ, 484, L25

Robinson, L., \& Wampler, E. J. 1972, PASP, 84, 161

Shortridge, K. 1988, The Figaro 2.4 Manual (Epping: Anglo-Australian Obs.)

Strom, S. E., Forte, J., Harris, W., Strom, K. M., Wells, D., \& Smith, M. 1981, ApJ, 245, 416

VandenBerg, D. A., Bolte, M., \& Stetson, P. B. 1996, ARA\&A, 36,461

van den Bergh, S. 1975, ARA\&A, 13, 217

Wheeler, J. C., Sneden, C., \& Truran, J. W. 1989, ARA\&A, 27, 279

Whitmore, B. C., Sparks, W. B., Lucas, R. A., Macchetto, F. D., \& Biretta, J. A. 1995, ApJ, 454, L73

Worthey, G. 1994, ApJS, 95, 107

Worthey, G., Faber, S. M., \& Gonzalez, J. J. 1992, ApJ, 398, 69

Worthey, G., \& Ottaviani, D. L. 1997, ApJS, 111, 445

Zinn, R. 1985, ApJ, 293, 424 